\title{
On the averaged quantum dynamics by white-noise Hamiltonians with and without dissipation
}

\author{
Werner Fischer ${ }^{1, *}$, Hajo Leschke ${ }^{1}$ and Peter Müller ${ }^{1,2}$ \\ ${ }^{1}$ Institut für Theoretische Physik, Universität Erlangen-Nürnberg, \\ Staudtstraße 7, 91058 Erlangen, Germany \\ ${ }^{2}$ Institut für Theoretische Physik, Georg-August-Universität, 37073 \\ Göttingen, Germany
}

Dedicated to Wolfgang Kundt on the occasion of his $65^{\text {th }}$ birthday

\begin{abstract}
Exact results are derived on the averaged dynamics of a class of random quantum-dynamical systems in continuous space. Each member of the class is characterized by a Hamiltonian which is the sum of two parts. While one part is deterministic, timeindependent and quadratic, the Weyl-Wigner symbol of the other part is a homogeneous Gaussian random field which is delta correlated in time, but smoothly correlated in position and momentum. The averaged dynamics of the resulting white-noise system is shown to be a monotone mixing increasing quantum-dynamical semigroup. Its generator is computed explicitly. Typically, in the course of time the mean energy of such a system grows linearly to infinity. In the second part of the paper an extended model is studied, which, in addition, accounts for dissipation by coupling the white-noise system linearly to a quantummechanical harmonic heat bath. It is demonstrated that, under suitable assumptions on the spectral density of the heat bath, the mean energy then saturates for long times.
\end{abstract}

Keywords. Random quantum-dynamical systems, white noise, dissipation.

\section{Introduction and summary of results}

Ever since Anderson (1958) one-particle quantum Hamiltonians with timeindependent random potentials have been intensively studied as models for disordered electronic systems (Bonch-Bruevich et al., 1984; Shklovskii and Efros, 1984; Lifshits et al., 1988; Carmona and Lacroix, 1990; Pastur and Figotin, 1992). In contrast, models with time-dependent random potentials have attracted less attention. They have been used, for example, to explain the diffusive behaviour of Frenkel excitons in molecular crystals (Haken and Reineker, 1972). Subsequently Ovchinnikov and Érikhman (1974), Madhukar and Post (1977) and Girvin and Mahan (1979) studied lattice models with time-dependent diagonal and off-diagonal random parts. Here the randomness enters via time-dependent fluctuating coupling coefficients and serves to

*New address: Siemens AG, Semiconductors, Balanstraße 73, 81541 München, Germany 
mimic the influence of collective excitations of the crystal. At high temperatures the coupling coefficients should be uncorrelated in time. More precisely, it is justified (Ovchinnikov and Érikhman, 1974) in this case to view them as realizations of a Gaussian white noise. One is then able to derive exact analytical results on the averaged dynamics of the model.

The simplifying feature of a vanishing correlation time was also exploited by Jayannavar and Kumar (1982). They were concerned with the averaged dynamics of a point particle moving in continuous space under the influence of a time-dependent Gaussian random potential which is uncorrelated in time, but smoothly correlated in position. Their main findings are a cubic longtime growth for the spatial spreading of states under the averaged dynamics and a linear increase in time of the mean energy. This behaviour is strikingly different from the diffusive spreading of states known from the aforementioned lattice models, which goes along with a saturating mean energy and may be interpreted in terms of Umklapp-processes. An extension of Jayannavar and Kumar (1982) to the case of an additionally present constant electric field is given in Jauho (1987).

A detailed study of models with uncorrelated time-dependent randomness is not only of interest for understanding specific problems in condensedmatter physics. Indeed, they are of relevance to many different branches of physics. Accordingly, the appearance of an additive white noise is not necessarily due to thermal fluctuations. Such models occur, for instance, in the debate on fundamental issues of quantum mechanics. In this context their averaged dynamics allows for a quantum-mechanical description of classical objects while still forbidding superpositions of macroscopically distinct state vectors, see for example Joos and Zeh (1985), Ghirardi et al. (1986), Joos (1987), Ghirardi et al. (1987), Bell (1987, p. 201), Ghirardi and Rimini (1990), Zurek (1991), Pearle and Squires (1994). In the realm of cosmology they are sought (Hawking, 1982; Banks et al., 1984; Hawking, 1984) to solve the information problem (Hawking, 1976) which arises by the evaporation of black holes. Furthermore they are met in classical kinetic theories (Spohn, 1980) and in quasi-classical Markovian transport theories (Markowich et al., 1990; Ferry and Grubin, 1995; Haug and Jauho, 1996).

Hence it is desirable to provide a systematic discussion of the stochastic dynamics of a rather general model with uncorrelated time-dependent randomness. This was initiated in our earlier paper (Fischer et al., 1994), where, as a first step, the averaged dynamics of such a model was studied. Since proofs of the results were only sketched there, it is one goal of the present paper to provide detailed proofs. The class of quantum-dynamical random systems which was introduced by Fischer et al. (1994) - see Model 2.1 in the next section - is characterized by a model Hamiltonian in continuous space which is the sum of a deterministic part and of a random part. Apart from allowing the deterministic part to be quadratic in both momentum and position, which may serve to describe the influence of a constant magnetic field, say, it is the other main feature of Fischer et al. (1994) that the random part may have fluctuations in both position and momentum. The fluctuations 
in momentum may be viewed as a continuum analogue of the off-diagonal random parts in the lattice models mentioned above or as a caricature for inelastic collisions of the particle. From the point of view of canonical mechanics they are natural anyway.

As to the mathematical description of random operators with fluctuations in position and momentum, one could think of applying a factor-ordering prescription to a random field on classical phase space. Therefore, and in order to clearly isolate quantum effects from classical ones even before taking expectation values, it is only natural not to use the conventional Hilbert-space formulation of quantum mechanics, but to represent the quantum system from the outset in phase space. In so doing, we choose the linear phasespace representation of Weyl, Wigner and Moyal, which amounts to a totally symmetric operator ordering when going back to Hilbert space.

As was already mentioned it is one goal of the present paper to provide detailed proofs of the results given in Fischer et al. (1994). This is done in Section 2. In Statement 2.2 we establish the semigroup property of the averaged dynamics of Model 2.1. Its irreversible character is described in Statement 2.4. Moreover, the averaged dynamics is positivity preserving and hence even a Markov semigroup, see Remark 2.6. Statement 2.7 is concerned with some geometrical properties of averaged time-evolved states and Statement 2.11 shows that in typical cases the mean energy of Model 2.1 grows linearly to infinity in the course of time. This unlimited increase of energy occurs because there is no mechanism built into Model 2.1 which causes energy to dissipate. It is therefore the second goal of the present paper to study also an extended version of Model 2.1 in which dissipation is incorporated, too. This is done in Section 3, where the white-noise system is linearly coupled to a quantum-mechanical heat bath of independent harmonic oscillators, see Model 3.1. The averaged dynamics of the total system, that is, white-noise system plus heat bath, is analyzed in Subsection 3.1 with the methods developed in Section 2. In Subsection 3.2 we eliminate the degrees of freedom of the heat bath assuming a thermal equilibrium state of the heat bath. Statement 3.12 at the end of Subsection 3.4, which is the main result of the second part of the paper, shows that the thus obtained reduced averaged dynamics of Model 3.1 acquires the desired dissipative features in the macroscopic limit of the heat bath provided its spectral density behaves suitably. The interplay of noise and dissipation then prevents the mean energy of the white-noise system from diverging in the long-time limit. The proof of this result requires some technical preparations which are dealt with in Subsections 3.3 and 3.4. Section 4 compiles some references concerning two related challenging problems which we do not address otherwise in this paper: fluctuations around the averaged dynamics and effects of a non-vanishing correlation time, that is, coloured-noise perturbations. Finally, for the convenience of the reader, the basic properties of the Weyl-Wigner-Moyal representation of quantum mechanics are presented in the Appendix. The reader who is interested in more details is referred to the (hopefully) up-to-date list of references. 


\section{The averaged dynamics by white-noise Hamiltonians}

In what follows, we are interested in the non-relativistic quantum dynamics of a single spinless point particle whose configuration space we choose to be, for simplicity, the Euclidean line $\mathbb{R}$. Following Weyl, Wigner and Moyal we will represent this quantum system in the two-dimensional symplectic phase space $\mathbb{R} \times \mathbb{R}$. The generalization to several Cartesian degrees of freedom is straightforward and will be dealt with on demand in Section 3.

\subsection{White-noise Hamiltonians}

In this subsection we define the basic model of this paper.

Model 2.1. Being the Weyl-Wigner symbol of a Hamiltonian operator, the Hamiltonian function

$H+N_{t}$,

also referred to as white-noise Hamiltonian in the sequel, defines a random quantum-dynamical system on phase space $\mathbb{R} \times \mathbb{R}$. The deterministic part $H$ of (2.1) is time independent and at most quadratic in momentum $p$ and position $q$. The time-dependent random part $N_{t}$ is a homogeneous Gaussian random field in $p, q$ and time $t$ with zero mean,

$\overline{N_{t}(p, q)}=0$,

and covariance function

$\overline{N_{t}(p, q) N_{t^{\prime}}\left(p^{\prime}, q^{\prime}\right)}=\delta\left(t-t^{\prime}\right) C\left(p-p^{\prime}, q-q^{\prime}\right)$.

Here we denote the stochastic average over randomness by an overbar, $\delta$ stands for the Dirac delta function and the phase-space part $C$ of the covariance function is assumed to be arbitrarily often differentiable.

The requirement for $H$ to be at most quadratic in position and momentum is necessary for deriving explicit results on the averaged dynamics of Model 2.1. On the other hand the time independence of $H$ and the homogeneity of $N_{t}$ are only assumed for simplicity. If one were interested in treating multiplicative noise (Habib, 1994), this could be managed, for example, by giving up the homogeneity assumption.

We would like to stress that in order for $C$ to be a covariance function it needs to be the Fourier transform of a positive measure on $\mathbb{R} \times \mathbb{R}$. This point is disregarded by Jayannavar (1993). Therefore it comes without surprise that some of his results are physically insignificant - especially those which are in contradiction to ours.

The dynamics generated by the white-noise Hamiltonian (2.1) is formally described by the stochastic quantum Liouville equation (Kubo , 1963)

$\partial_{t} w_{t}=-\left[H, w_{t}\right]-\left[N_{t}, w_{t}\right]$ 
with initial condition $w_{0}=w$. Here $w$ is the Wigner density representing the initial state and $[\bullet, \bullet]$ denotes the Moyal bracket (A7) of two phasespace functions. Note that the first Moyal bracket $\left[H, w_{t}\right]$ in $(2.4)$ is in fact a Poisson bracket due to the quadratic nature of $H$, cf. Remark A.3. Being a stochastic partial differential equation, (2.4) has to be supplemented by a prescription of how to interpret the second Moyal bracket on its right-hand side, which involves the distribution-valued white noise $N_{t}$. We choose a prescription which is quite natural on physical grounds: The time change of a realistic physical system is governed by driving forces with a non-zero correlation time. Theoretical models based on stochastic processes which are uncorrelated in time may only be used successfully if the correlation time of the actual driving forces is much smaller than any other time scale inherent to the system. Therefore we interpret (2.4) in the Stratonovich sense whichas is supported by the Wong-Zakai-like theorems in Horsthemke and Lefever (1984, p. 101), Karatzas and Shreve (1988, Chap. 5.2.D) and Brzeźniak and Flandoli (1995) - corresponds to considering the white-noise limit $\tau \searrow 0$ of the solution $w_{t}^{(\tau)}$ of the regularized initial-value problem

$\partial_{t} w_{t}^{(\tau)}=-\left[H, w_{t}^{(\tau)}\right]-\left[N_{t}^{(\tau)}, w_{t}^{(\tau)}\right]$,

$w_{0}^{(\tau)}=w$.

In (2.5) we have replaced the Gaussian white noise $N_{t}$ by the Gaussian coloured noise $N_{t}^{(\tau)}$ which vanishes in mean, $\overline{N_{t}^{(\tau)}(p, q)}=0$, and is correlated according to

$\overline{N_{t}^{(\tau)}(p, q) N_{t^{\prime}}^{(\tau)}\left(p^{\prime}, q^{\prime}\right)}=g^{(\tau)}\left(t-t^{\prime}\right) C\left(p-p^{\prime}, q-q^{\prime}\right)$,

where $g^{(\tau)}$ is a correlation function that approaches the Dirac delta function in the limit of vanishing correlation time $\tau \searrow 0$. For times $t \geq 0$ the solution of (2.5) can be conveniently expressed in terms of a time-ordered exponential

$$
\begin{aligned}
w_{t}^{(\tau)}= & w+\sum_{n=1}^{\infty}(-1)^{n} \int_{0}^{t} \mathrm{~d} s_{n} \int_{0}^{s_{n}} \mathrm{~d} s_{n-1} \cdots \int_{0}^{s_{2}} \mathrm{~d} s_{1} \\
& \times\left[H+N_{s_{n}}^{(\tau)},\left[H+N_{s_{n-1}}^{(\tau)}, \ldots,\left[H+N_{s_{1}}^{(\tau)}, w\right] \ldots\right]\right] \\
= & =\exp \left\{-\int_{0}^{t} \mathrm{~d} s\left[H+N_{s}^{(\tau)}, \bullet\right]\right\} w .
\end{aligned}
$$

We remark that the corresponding solution for times $t \leq 0$ requires the time ordering to be reversed.

\subsection{The averaged dynamics}

The averaged dynamics generated by a white-noise Hamiltonian is the mapping $\mathcal{T}_{t}$ defined by

$w \mapsto \mathcal{T}_{t} w:=\overline{w_{t}}$, 
where $\overline{w_{t}}(p, q):=\overline{w_{t}(p, q)}$ denotes the stochastic mean of the solution of $(2.4)$ in the Stratonovich interpretation. According to the preceding subsection, $\mathcal{T}_{t}$ can be expressed as the limit

$$
\mathcal{T}_{t}=\lim _{\tau \searrow 0} \overline{\exp \left\{-\int_{0}^{t} \mathrm{~d} s\left[H+N_{s}^{(\tau)}, \bullet\right]\right\}}, \quad t \geq 0 .
$$

The salient feature of the white-noise limit is that it allows to describe the averaged dynamics explicitly.

Statement 2.2. The averaged dynamics of $H+N_{t}$ is a semigroup of linear operators

$\mathcal{T}_{t}=\exp \{-t(\mathcal{L}+\mathcal{N})\}$

Its generator consists of the Liouvillian

$\mathcal{L}:=[H, \bullet]=\left(\partial_{p} H\right) \partial_{q}-\left(\partial_{q} H\right) \partial_{p}$

associated with the deterministic part $H$ of the Hamiltonian and of the perturbation

$\mathcal{N}:=\hbar^{-2}\left\{C(0,0)-C\left(-\mathrm{i} \hbar \partial_{q}, \mathrm{i} \hbar \partial_{p}\right)\right\}$

which depends only on the stochastic properties of the random part $N_{t}$.

Proof. To perform the stochastic average in (2.9) it is advantageous (Kubo, $1962, \S 7(5))$ to isolate the unperturbed time evolution generated by $\mathcal{L}$ in passing to the Dirac-Dyson representation of the time-evolution operator

$\overleftarrow{\exp }\left\{-\int_{0}^{t} \mathrm{~d} s\left(\mathcal{L}+\left[N_{s}^{(\tau)}, \cdot\right]\right)\right\}=\mathrm{e}^{-t \mathcal{L}} \overleftarrow{\exp }\left\{-\int_{0}^{t} \mathrm{~d} s \mathrm{e}^{s \mathcal{L}}\left[N_{s}^{(\tau)}, \bullet\right] \mathrm{e}^{-s \mathcal{L}}\right\}$.

Next we introduce the family of Gaussian-distributed random complex measures $\tilde{N}_{t}^{(\tau)}(\mathrm{d} p \mathrm{~d} q)$ on phase space arising in the spectral representation (Doob, 1964, Chap. 11, § 4)

$N_{t}^{(\tau)}(p, q)=: \int_{\mathbb{R} \times \mathbb{R}} \tilde{N}_{t}^{(\tau)}\left(\mathrm{d} p^{\prime} \mathrm{d} q^{\prime}\right) \mathrm{e}^{\mathrm{i}\left(p q^{\prime}-q p^{\prime}\right) / \hbar}$

of $N_{t}^{(\tau)}$. They have zero mean, $\overline{\tilde{N}_{t}^{(\tau)}(\mathrm{d} p \mathrm{~d} q)}=0$, and the covariance measure $\overline{\tilde{N}_{t}^{(\tau)}(\mathrm{d} p \mathrm{~d} q) \tilde{N}_{t^{\prime}}^{(\tau)}\left(\mathrm{d} p^{\prime} \mathrm{d} q^{\prime}\right)}=g^{(\tau)}\left(t-t^{\prime}\right) \tilde{C}(\mathrm{~d} p \mathrm{~d} q) \delta_{-p,-q}\left(\mathrm{~d} p^{\prime} \mathrm{d} q^{\prime}\right)$.

Here $\delta_{-p,-q}\left(\mathrm{~d} p^{\prime} \mathrm{d} q^{\prime}\right)=\delta\left(p^{\prime}+p\right) \delta\left(q^{\prime}+q\right) \mathrm{d} p^{\prime} \mathrm{d} q^{\prime}$ denotes the Dirac measure with support $(-p,-q) \in \mathbb{R} \times \mathbb{R}$. The covariance function $C$ appears as the symplectic Fourier transform

$C(p, q)=\int_{\mathbb{R} \times \mathbb{R}} \tilde{C}\left(\mathrm{~d} p^{\prime} \mathrm{d} q^{\prime}\right) \mathrm{e}^{\mathrm{i}\left(p q^{\prime}-q p^{\prime}\right) / \hbar}$ 
of the measure $\tilde{C}$ on $\mathbb{R} \times \mathbb{R}$. Inserting (2.14) into the definition (A7) of the Moyal bracket one obtains

$\left[N_{s}^{(\tau)}, \bullet\right]=\mathrm{i} \int_{\mathbb{R} \times \mathbb{R}} \tilde{N}_{s}^{(\tau)}\left(\mathrm{d} p^{\prime} \mathrm{d} q^{\prime}\right) \mathcal{F}\left(p^{\prime}, q^{\prime}\right)$.

Here we have introduced the operator-valued function

$\mathbb{R} \times \mathbb{R} \ni\left(p^{\prime}, q^{\prime}\right) \mapsto \mathcal{F}\left(p^{\prime}, q^{\prime}\right):=\hbar^{-1} \mathrm{e}^{\mathrm{i}\left(p q^{\prime}-q p^{\prime}\right) / \hbar}\left(\mathcal{V}_{p^{\prime} / 2, q^{\prime} / 2}-\mathcal{V}_{-p^{\prime} / 2,-q^{\prime} / 2}\right)$

In (2.18) $p$ and $q$ play the rôle of the canonical coordinate functions on phase space and $\mathcal{V}_{p^{\prime}, q^{\prime}}:=\mathrm{e}^{p^{\prime} \partial_{p}+q^{\prime} \partial_{q}}$ is the translation operator by $\left(p^{\prime}, q^{\prime}\right)$. In other words, $\mathcal{F}\left(p^{\prime}, q^{\prime}\right)$ acts on a phase-space function $f$ according to

$$
\begin{aligned}
\left(\mathcal{F}\left(p^{\prime}, q^{\prime}\right) f\right)(p, q)=\hbar^{-1} \mathrm{e}^{\mathrm{i}\left(p q^{\prime}-q p^{\prime}\right) / \hbar}\{ & f\left(p+p^{\prime} / 2, q+q^{\prime} / 2\right) \\
& \left.-f\left(p-p^{\prime} / 2, q-q^{\prime} / 2\right)\right\} .
\end{aligned}
$$

With these preparations and writing $\mathcal{F}_{s}\left(p^{\prime}, q^{\prime}\right):=\mathrm{e}^{s \mathcal{L}} \mathcal{F}\left(p^{\prime}, q^{\prime}\right) \mathrm{e}^{-s \mathcal{L}}$ for the Dirac picture of $\mathcal{F}\left(p^{\prime}, q^{\prime}\right)$, the averaged dynamics can be cast into the form

$$
\mathcal{T}_{t}=\mathrm{e}^{-t \mathcal{L}} \lim _{\tau \searrow 0} \overline{\exp \left\{-\mathrm{i} \int_{0}^{t} \mathrm{~d} s \int_{\mathbb{R} \times \mathbb{R}} \tilde{N}_{s}^{(\tau)}\left(\mathrm{d} p^{\prime} \mathrm{d} q^{\prime}\right) \mathcal{F}_{s}\left(p^{\prime}, q^{\prime}\right)\right\}} .
$$

To perform the stochastic average in (2.20) we appeal to the characteristic functional of Gaussian random variables and conclude that

$$
\begin{aligned}
\mathcal{T}_{t}= & \mathrm{e}^{-t \mathcal{L}} \lim _{\tau \searrow 0} \overleftarrow{\exp }\left\{-\frac{1}{2} \int_{0}^{t} \mathrm{~d} s \int_{0}^{t} \mathrm{~d} s^{\prime} g^{(\tau)}\left(s-s^{\prime}\right)\right. \\
& \left.\times \int_{\mathbb{R} \times \mathbb{R}} \tilde{C}\left(\mathrm{~d} p^{\prime} d q^{\prime}\right) \mathcal{F}_{s}\left(-p^{\prime},-q^{\prime}\right) \mathcal{F}_{s^{\prime}}\left(p^{\prime}, q^{\prime}\right)\right\} \\
= & \mathrm{e}^{-t \mathcal{L}} \overleftarrow{\exp }\left\{-\frac{1}{2} \int_{0}^{t} \mathrm{~d} s \int_{\mathbb{R} \times \mathbb{R}} \tilde{C}\left(\mathrm{~d} p^{\prime} d q^{\prime}\right) \mathcal{F}_{s}\left(-p^{\prime},-q^{\prime}\right) \mathcal{F}_{s}\left(p^{\prime}, q^{\prime}\right)\right\} .
\end{aligned}
$$

Furthermore one has

$$
\begin{aligned}
\int_{\mathbb{R} \times \mathbb{R}} \tilde{C}\left(\mathrm{~d} p^{\prime} d q^{\prime}\right) & \mathcal{F}\left(-p^{\prime},-q^{\prime}\right) \mathcal{F}\left(p^{\prime}, q^{\prime}\right) \\
& =\hbar^{-2} \int_{\mathbb{R} \times \mathbb{R}} \tilde{C}\left(\mathrm{~d} p^{\prime} d q^{\prime}\right)\left(2-\mathcal{V}_{p^{\prime}, q^{\prime}}-\mathcal{V}_{-p^{\prime},-q^{\prime}}\right) \\
& =2 \hbar^{-2}\left\{C(0,0)-C\left(-\mathrm{i} \hbar \partial_{q}, \mathrm{i} \hbar \partial_{p}\right)\right\} \\
& =2 \mathcal{N}
\end{aligned}
$$

because $C$ is an even function. Hence

$$
\mathcal{T}_{t}=\mathrm{e}^{-t \mathcal{L}} \overleftarrow{\exp }\left\{-\int_{0}^{t} \mathrm{~d} s \mathrm{e}^{s \mathcal{L}} \mathcal{N} \mathrm{e}^{-s \mathcal{L}}\right\}=\mathrm{e}^{-t(\mathcal{L}+\mathcal{N})},
$$

and the proof is complete. 
Remark 2.3. It follows from Statement 2.2 by a differentiation with respect to time that the averaged state $\overline{w_{t}}$ obeys the linear integro-differential equation

$$
\begin{gathered}
\partial_{t} \overline{w_{t}}(p, q)=\left[\overline{w_{t}}, H\right](p, q)+\hbar^{-2} \int_{\mathbb{R} \times \mathbb{R}} \tilde{C}\left(\mathrm{~d} p^{\prime} \mathrm{d} q^{\prime}\right)\left\{\overline{w_{t}}\left(p+p^{\prime}, q+q^{\prime}\right)\right. \\
\left.-\overline{w_{t}}(p, q)\right\}
\end{gathered}
$$

with initial condition $\overline{w_{0}}=w$. Eq. (2.24) is a generalization of the main result of Jayannavar and Kumar (1982). In the special case of a free deterministic part, $H=p^{2} / 2 m$, and a momentum-independent noise, that is, when $C$ does not depend on $p$, it reduces to an equation which is equivalent to Eq. (8) in Jayannavar and Kumar (1982). The latter is derived there by directly averaging the von Neumann equation with the help of a partial integration with respect to the Gaussian probability measure (Furutsu, 1963; Donsker, 1964; Novikov, 1964), see also Rytov et al. (1989, p. 32) or Glimm and Jaffe (1987, Thm. 6.3.1). The present approach is more direct and appears to provide a better starting point towards a generalization to the case of non-vanishing correlation times, that is, coloured noise. Note also that the subsequent treatment of Eq. (8) in Jayannavar and Kumar (1982) is restricted to a pure initial state which, in Weyl-Wigner language, is represented by a Gaussian Wigner density $w$.

Being the stochastic average of a Hamiltonian dynamics the semigroup $\mathcal{T}_{t}$ is completely positive and thus provides an example of a quantum-dynamical semigroup in the sense of Bausch (1966), Gorini et al. (1976), Lindblad (1976), Spohn (1980), Alicki and Lendi (1987) or Streater (1995). Quantumdynamical equations similar to (2.24) are discussed in very different branches of physics. One reason for this is revealed by

Statement 2.4. The averaged dynamics is monotone mixing increasing in the sense that

$\partial_{t}\left\langle\mathcal{T}_{t} w, \mathcal{T}_{t} w\right\rangle \leq 0$

for all $t>0$ and all Wigner densities $w$. Here we use the notation $\langle\cdot, \cdot\rangle$ for the standard scalar product (A4) of phase-space functions. In (2.25) equality holds if and only if $C$ is a constant.

Proof. Taking the scalar product of (2.24) with $\overline{w_{t}}$ and observing $\left\langle\overline{w_{t}},\left[\overline{w_{t}}, H\right]\right\rangle=0$, which follows from a partial integration, we get

$\left\langle\overline{w_{t}}, \partial_{t} \overline{w_{t}}\right\rangle=\hbar^{-2} \int_{\mathbb{R} \times \mathbb{R}} \tilde{C}\left(\mathrm{~d} p^{\prime} \mathrm{d} q^{\prime}\right)\left\{\left\langle\overline{w_{t}}, \mathcal{V}_{p^{\prime}, q^{\prime}} \overline{w_{t}}\right\rangle-\left\langle\overline{w_{t}}, \overline{w_{t}}\right\rangle\right\}$.

Due to the positivity of the measure $\tilde{C}$, inequality (2.25) now follows from an application of the Cauchy-Schwarz inequality

$\left\langle\overline{w_{t}}, \mathcal{V}_{p^{\prime}, q^{\prime}} \overline{w_{t}}\right\rangle \leq\left\langle\overline{w_{t}}, \overline{w_{t}}\right\rangle^{1 / 2}\left\langle\mathcal{V}_{p^{\prime}, q^{\prime}} \overline{w_{t}}, \mathcal{V}_{p^{\prime}, q^{\prime}} \overline{w_{t}}\right\rangle^{1 / 2}=\left\langle\overline{w_{t}}, \overline{w_{t}}\right\rangle$ 
To obtain equality in (2.25) it is necessary and sufficient that there is equality in (2.27) for $\tilde{C}$-almost all $\left(p^{\prime}, q^{\prime}\right)$. Hence, it is necessary and sufficient that the functions $\overline{w_{t}}$ and $\mathcal{V}_{p^{\prime}, q^{\prime}} \overline{w_{t}}$ are linearly dependent for $\tilde{C}$-almost all $\left(p^{\prime}, q^{\prime}\right)$. In this case there are constants $\lambda_{p^{\prime}, q^{\prime}}$ with $\mathcal{V}_{p^{\prime}, q^{\prime}} \overline{w_{t}}=\lambda_{p^{\prime}, q^{\prime}} \overline{w_{t}}$. Normalization $\left\langle 1, \mathcal{V}_{p^{\prime}, q^{\prime}} \overline{w_{t}}\right\rangle=\left\langle 1, \overline{w_{t}}\right\rangle$ requires $\lambda_{p^{\prime}, q^{\prime}}=1$. For $\left(p^{\prime}, q^{\prime}\right) \neq(0,0)$ this implies periodicity of the Wigner density $\overline{w_{t}}$ which is in contradiction to its squareintegrability $\left\langle\overline{w_{t}}, \overline{w_{t}}\right\rangle \leq(2 \pi \hbar)^{-1}<\infty$, cf. Statement A.5. To summarize, the Wigner densities $\overline{w_{t}}$ and $\mathcal{V}_{p^{\prime}, q^{\prime}} \overline{w_{t}}$ are linearly dependent if and only if $\left(p^{\prime}, q^{\prime}\right)=(0,0)$ which in turn allows for equality in (2.25) if and only if $\tilde{C}$ has support $\{(0,0)\}$.

Statement 2.4 can be reformulated as $\partial_{t} S_{2}\left(\mathcal{T}_{t} w\right) \geq 0$, where $S_{2}(w):=$ $-\ln (2 \pi \hbar\langle w, w\rangle)$ is the Rényi entropy of order two of the quantum state (Jumarie, 1990). Similar to the von Neumann entropy, $S_{2}$ reflects the degree of mixing of the quantum state (Thirring, 1983, (2.2,3)) and, by Jensen's inequality, it is a non-negative lower bound to the former. The average over randomness has turned the fully reversible quantum Liouville equation (2.4) into the "master equation" (2.24) with coherence-destructing irreversible behaviour.

So far we have not made use of the fact that the deterministic part $H$ of the Hamiltonian is at most quadratic in $p$ and $q$. The next result however relies crucially on it.

Statement 2.5. The averaged dynamics admits the representation

$\mathcal{T}_{t}=\mathrm{e}^{-t \mathcal{L}} \exp \left\{-\hbar^{-2} \int_{0}^{t} \mathrm{~d} s\left(C(0,0)-C\left(-\mathrm{i} \hbar \mathcal{K}_{s}, \mathrm{i} \hbar \mathcal{X}_{s}\right)\right)\right\}$.

The coefficients of the first-order differential operators

$\begin{aligned} \mathcal{K}_{s} & :=\left(\partial_{q} \mathrm{e}^{-s \mathcal{L}} p\right) \partial_{p}+\left(\partial_{q} \mathrm{e}^{-s \mathcal{L}} q\right) \partial_{q}, \\ \mathcal{X}_{s} & :=\left(\partial_{p} \mathrm{e}^{-s \mathcal{L}} p\right) \partial_{p}+\left(\partial_{p} \mathrm{e}^{-s \mathcal{L}} q\right) \partial_{q}\end{aligned}$

depend only on time and not on position and momentum. They are determined by the classical phase-space trajectories generated by $H$.

Note that there is no time ordering in (2.28) because the four operators $\mathcal{K}_{s}$, $\mathcal{K}_{s^{\prime}}, \mathcal{X}_{s}, \mathcal{X}_{s^{\prime}}$ commute pairwise due to the quadratic nature of $H$. Statement 2.5 is important because it allows to derive explicit results on the averaged dynamics $\mathcal{T}_{t}$.

Proof of Statement 2.5. Thanks to (2.23) it only remains to show that

$\mathrm{e}^{s \mathcal{L}} \mathcal{N} \mathrm{e}^{-s \mathcal{L}}=\hbar^{-2}\left(C(0,0)-C\left(-\mathrm{i} \hbar \mathcal{K}_{s}, \mathrm{i} \hbar \mathcal{X}_{s}\right)\right)$.

But when applying both sides of (2.30) to a phase-space function, this identity follows from the chain rule and from observing that the phase-space trajectories $\left\{\left(e^{t \mathcal{L}} p\right)\left(p^{\prime}, q^{\prime}\right),\left(e^{t \mathcal{L}} q\right)\left(p^{\prime}, q^{\prime}\right)\right\}_{t \geq 0}$ are linear functions in the initial data $\left(p^{\prime}, q^{\prime}\right)$ due to the quadratic nature of $H$. 
To gain some more insight into the behaviour of $\mathcal{T}_{t}$ define the phase-space function

$\tilde{P}_{t}(p, q):=\mathrm{e}^{-t C(0,0) / \hbar^{2}} \exp \left\{\hbar^{-2} \int_{0}^{t} \mathrm{~d} s\left(C \circ \mathrm{J}_{-s}^{\dagger}\right)(p, q)\right\}$,

where

$\mathrm{J}_{t}:=\left(\begin{array}{ll}\partial_{p} \mathrm{e}^{t \mathcal{L}} p & \partial_{q} \mathrm{e}^{t \mathcal{L}} p \\ \partial_{p} \mathrm{e}^{\mathrm{L}_{q}} & \partial_{q} \mathrm{e}^{t \mathcal{L}} q\end{array}\right)$

is the Jacobian of the flow $\mathrm{e}^{t \mathcal{L}}$ on phase space and $\mathrm{J}_{t}^{\dagger}:=\left(\begin{array}{cc}0 & -1 \\ 1 & 0\end{array}\right) \mathrm{J}_{t}^{\top}\left(\begin{array}{cc}0 & 1 \\ -1 & 0\end{array}\right)$ is its adjoint with respect to the canonical symplectic form $\left((p, q),\left(p^{\prime}, q^{\prime}\right)\right) \mapsto$ $p q^{\prime}-q p^{\prime}$. The superscript ${ }^{\top}$ denotes the transposition of matrices and the circle $\circ$ denotes the composition of mappings. Because of

$$
\left(\begin{array}{c}
-\mathrm{i} \hbar \mathcal{K}_{s} \\
\mathrm{i} \hbar \mathcal{X}_{s}
\end{array}\right)=J_{-s}^{\dagger}\left(\begin{array}{c}
-\mathrm{i} \hbar \partial_{q} \\
\mathrm{i} \hbar \partial_{p}
\end{array}\right)
$$

one can write $\mathcal{T}_{t}=\mathrm{e}^{-t \mathcal{L}} \tilde{P}_{t}\left(-\mathrm{i} \hbar \partial_{q}, \mathrm{i} \hbar \partial_{p}\right)$. Now observe that $\tilde{P}_{t}$ is a characteristic function, that is, the Fourier transform of a measure $P_{t}$ on phase space

$\tilde{P}_{t}(p, q)=: \int_{\mathbb{R} \times \mathbb{R}} P_{t}\left(\mathrm{~d} p^{\prime} \mathrm{d} q^{\prime}\right) \mathrm{e}^{\mathrm{i}\left(p q^{\prime}-q p^{\prime}\right) / \hbar}$.

This is true because $\hbar^{-2} \int_{0}^{t} \mathrm{~d} s C \circ \mathrm{J}_{-s}^{\dagger}$ is a characteristic function and because sums and products of characteristic functions are again characteristic functions. In fact, $P_{t}$ is even a probability measure due to $\tilde{P}_{t}(0,0)=1$. Thus we conclude

$$
\mathcal{T}_{t}=\mathrm{e}^{-t \mathcal{L}} \int_{\mathbb{R} \times \mathbb{R}} P_{t}\left(\mathrm{~d} p^{\prime} \mathrm{d} q^{\prime}\right) \mathcal{V}_{p^{\prime}, q^{\prime}} .
$$

Remark 2.6. The result (2.35) shows that the averaged dynamics is also positivity preserving and thus even a Markov semigroup. In particular, it maps classical states, that is, probability densities on $\mathbb{R} \times \mathbb{R}$, to classical states. Eq. (2.24) appears as the associated kinetic equation, which - up to the drift term arising from $H$-may be viewed upon as a linear Boltzmann equation with a homogeneous stochastic kernel (Lasota and Mackey, 1994; Reed and Simon, 1979, Chap. XI.12).

Since the covariance function $C$ of the random field is an even function, all odd moments of the probability measure $P_{t}$ vanish. The covariance matrix of $P_{t}$

$$
\begin{aligned}
C_{t}: & =\int_{\mathbb{R} \times \mathbb{R}} P_{t}(\mathrm{~d} p \mathrm{~d} q)\left(\begin{array}{cc}
p^{2} & p q \\
p q & q^{2}
\end{array}\right)=-\hbar^{2}\left(\left(\partial^{2} \tilde{P}_{t}\right)(0,0)\right)^{\dagger} \\
& =\int_{0}^{t} \mathrm{~d} s \mathrm{~J}_{-s}\left(\left(-\partial^{2} C\right)(0,0)\right)^{\dagger} \mathrm{J}_{-s}^{\top}
\end{aligned}
$$


is monotone increasing in time,

$0 \leq \mathrm{C}_{t^{\prime}} \leq \mathrm{C}_{t}, \quad 0 \leq t^{\prime} \leq t$.

In (2.36) we have used the symbol $\partial^{2}$ for the Hessian of second-order partial derivatives. For later purpose it is convenient to introduce the non-negative matrix of diffusion coefficients

$\mathrm{D}:=\left(\begin{array}{cc}D_{0,2} & \frac{1}{2} D_{1,1} \\ \frac{1}{2} D_{1,1} & D_{2,0}\end{array}\right)=-\frac{1}{2}\left(\left(\partial^{2} C\right)(0,0)\right)^{\dagger} \geq 0$

whose entries

$D_{\mu, \nu}:=\left(\left(-i \partial_{p}\right)^{\mu}\left(i \partial_{q}\right)^{\nu} C\right)(0,0) /(\mu ! \nu !)$

are given by the curvature of the covariance function at the origin. Thus one can also write

$\mathrm{C}_{t}=2 \int_{0}^{t} \mathrm{~d} s \mathrm{~J}_{-s} \mathrm{D} \mathrm{J}_{-s}^{\mathrm{T}}$.

It is clear from the Riemann-Lebesgue lemma that the probability measure $P_{t}$ cannot be absolutely continuous with respect to Lebesgue measure $\mathrm{d} p \mathrm{~d} q$. The following decomposition helps to decide whether $P_{t}$ possesses an absolutely continuous component.

Statement 2.7. The averaged dynamics of an initial state $w$

$$
\begin{aligned}
\left(\mathcal{T}_{t} w\right)(p, q)= & \mathrm{e}^{-t C(0,0) / \hbar^{2}} w\left(\mathrm{e}^{-t \mathcal{L}} p, \mathrm{e}^{-t \mathcal{L}} q\right) \\
& +\int_{\mathbb{R} \times \mathbb{R}} Q_{t}\left(\mathrm{~d} p^{\prime} \mathrm{d} q^{\prime}\right) w\left(p^{\prime}+\mathrm{e}^{-t \mathcal{L}} p, q^{\prime}+\mathrm{e}^{-t \mathcal{L}} q\right)
\end{aligned}
$$

decomposes into an exponentially decaying contribution of the unperturbed dynamics and into a contribution where the initial state is first smeared out by the finite measure $Q_{t}$ and then subjected to the unperturbed dynamics. The measure $Q_{t}$ is defined by its characteristic function

$\tilde{Q}_{t}:=\tilde{P}_{t}-\mathrm{e}^{-t C(0,0) / \hbar^{2}}$

and is normalized to $\tilde{Q}_{t}(0,0)=1-\mathrm{e}^{-t C(0,0) / \hbar^{2}}$. All higher moments of $Q_{t}$ coincide with those of $P_{t}$. A sufficient condition for $Q_{t}$ to have an integrable and continuous density with respect to Lebesgue measure is the integrability of $C$. The density is then bounded by

$0 \leq \frac{Q_{t}(\mathrm{~d} p \mathrm{~d} q)}{\mathrm{d} p \mathrm{~d} q} \leq \frac{t}{\left(2 \pi \hbar^{2}\right)^{2}} \int_{\mathbb{R} \times \mathbb{R}} \mathrm{d} p^{\prime} \mathrm{d} q^{\prime}\left|C\left(p^{\prime}, q^{\prime}\right)\right|$.

Necessary conditions for the existence of a density are the strict positivity of the covariance matrix, $\mathrm{C}_{t}>0$, and the vanishing of the function $(p, q) \mapsto$ $\int_{0}^{t} \mathrm{~d} s\left(C \circ \mathrm{J}_{-s}^{\dagger}\right)(p, q)$ at infinity. 


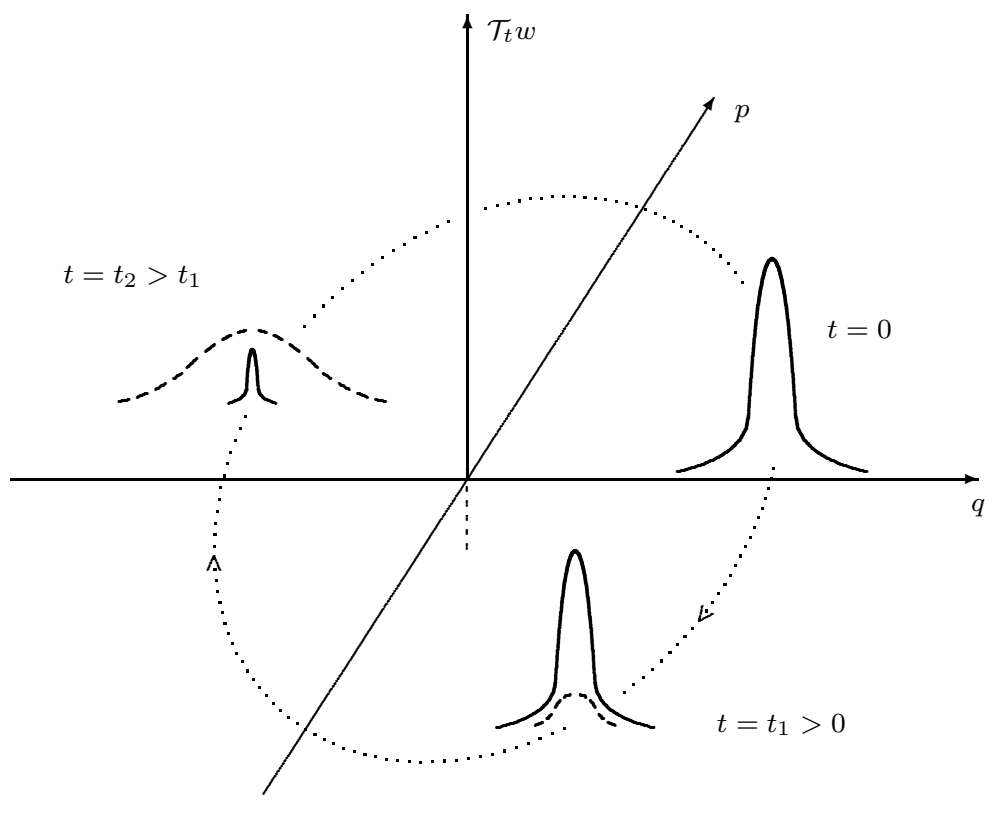

Fig. 1. Illustration of the averaged dynamics of a Wigner density in phase space. The deterministic part of the Hamiltonian has been chosen to be that of a harmonic oscillator. The solid (dashed) contribution symbolizes the first (second) term in the decomposition (2.41).

Figure 1 illustrates the situation described in Statement 2.7 for the case where $H$ is the Hamiltonian of a harmonic oscillator and $w$ a non-negative initial Wigner density at time $t=0$. The dotted ellipse represents one phase-space trajectory of $H$. The averaged state $\mathcal{T}_{t} w$ at times $t_{1}>0$ and $t_{2}>t_{1}$ is obtained by summing up the solid and the dashed contribution which symbolize the first and second term in the decomposition (2.41), respectively. Accordingly, the weight of the solid contribution to $\mathcal{T}_{t} w$ decreases exponentially in time, whereas that of the dashed one increases to one. In addition, the width of the dashed contribution increases in time. The sum of the volumes enclosed underneath is always one.

Proof of Statement 2.7. The arguments which established that $\tilde{P}_{t}$ is a characteristic function apply to $\tilde{Q}_{t}$ as well. Hence, $Q_{t}$ is a finite measure on $\mathbb{R} \times \mathbb{R}$. According to Bauer $\left(1996, \S 23\right.$, Problem 10) the integrability of $\tilde{Q}_{t}$ is sufficient to guarantee the existence and continuity of the Lebesgue density $Q_{t}(\mathrm{~d} p \mathrm{~d} q) / \mathrm{d} p \mathrm{~d} q$. But the inequality $\left|\mathrm{e}^{z}-1\right| \leq|z| \mathrm{e}^{y}$, which is valid for all $|z| \leq y$, shows that this condition is fulfilled if $C$ is integrable. Here, we have also used the fact that phase-space volume is conserved by Hamiltonian flows. The upper bound in (2.43) then follows from the Fourier inversion for- 
mula. The second of the two necessary conditions is just the content of the Riemann-Lebesgue lemma. As to the first one we remark that if it did not hold, there would exist a pair $\left(p^{\prime}, q^{\prime}\right)$ such that $\int_{\mathbb{R} \times \mathbb{R}} Q_{t}(\mathrm{~d} p \mathrm{~d} q)\left(p^{\prime} q-q^{\prime} p\right)^{2}=0$. But this implies that $Q_{t}$ is supported on a set of Lebesgue measure zero.

\subsection{The classical limit}

One main advantage of a phase-space representation of quantum mechanics is that the classical limit of a quantum system may be studied with little efforts. We denote the averaged dynamics of the classical white-noise system by

$\mathcal{T}_{t, \mathrm{cl}}:=\lim _{\hbar \searrow 0} \mathcal{T}_{t}$

and conclude

Statement 2.8. Suppose that the deterministic part $H$ of the Hamiltonian and the covariance function $C$ do not depend on $\hbar$.

Then the averaged dynamics of the classical white-noise system

i) is a semigroup of linear operators

$$
\mathcal{T}_{t, \mathrm{cl}}=\exp \left\{-t\left(\mathcal{L}+\mathcal{N}_{\mathrm{cl}}\right)\right\}=\mathrm{e}^{-t \mathcal{L}} \exp \left\{\frac{1}{2}\left(\partial_{p}, \partial_{q}\right) \mathrm{C}_{t}\left(\begin{array}{c}
\partial_{p} \\
\partial_{q}
\end{array}\right)\right\}
$$

with generator $\mathcal{L}+\mathcal{N}_{\mathrm{cl}}$, where

$$
\mathcal{N}_{\mathrm{cl}}:=-\left(\partial_{p}, \partial_{q}\right) \mathrm{D}\left(\begin{array}{c}
\partial_{p} \\
\partial_{q}
\end{array}\right) \text {. }
$$

The covariance matrix $\mathrm{C}_{t}$ and the matrix $\mathrm{D}$ of diffusion coefficients were introduced in (2.36) and (2.38), respectively.

ii) maps an initial probability density $\rho$ on phase space to the probability density $\overline{\rho_{t}}:=\mathcal{T}_{t, \mathrm{cl}} \rho$ which obeys the Fokker-Planck equation

$$
\partial_{t} \overline{\rho_{t}}=\left(\left(\partial_{q} H\right) \partial_{p}-\left(\partial_{p} H\right) \partial_{q}\right) \overline{\rho_{t}}+\left(\partial_{p}, \partial_{q}\right) \mathrm{D}\left(\begin{array}{c}
\partial_{p} \\
\partial_{q}
\end{array}\right) \overline{\rho_{t}}
$$

with initial condition $\overline{\rho_{0}}=\rho$. The solution of (2.47) is obtained by smearing out $\rho$ with the Gaussian probability measure $P_{t, \mathrm{cl}}$ on phase space, which is defined by its characteristic function

$$
\exp \left\{-\frac{1}{2}(x, k) \mathrm{C}_{t}\left(\begin{array}{l}
x \\
k
\end{array}\right)\right\}=: \int_{\mathbb{R} \times \mathbb{R}} P_{t, \mathrm{cl}}\left(\mathrm{d} p^{\prime} \mathrm{d} q^{\prime}\right) \mathrm{e}^{-\mathrm{i}\left(x p^{\prime}+k q^{\prime}\right)},
$$

and subjecting the result to the time evolution of $H$

$$
\overline{\rho_{t}}(p, q)=\int_{\mathbb{R} \times \mathbb{R}} P_{t, \mathrm{cl}}\left(\mathrm{d} p^{\prime} \mathrm{d} q^{\prime}\right) \rho\left(p^{\prime}+\mathrm{e}^{-t \mathcal{L}} p, q^{\prime}+\mathrm{e}^{-t \mathcal{L}} q\right) .
$$


The measure $P_{t, \mathrm{cl}}$ has zero mean and $\mathrm{C}_{t}$ as its covariance matrix. It possesses the Lebesgue density

$\frac{P_{t, \mathrm{cl}}(\mathrm{d} p \mathrm{~d} q)}{\mathrm{d} p \mathrm{~d} q}=\left(\operatorname{det}\left(2 \pi \mathrm{C}_{t}\right)\right)^{-1 / 2} \exp \left\{-\frac{1}{2}(p, q) \mathrm{C}_{t}^{-1}\left(\begin{array}{l}p \\ q\end{array}\right)\right\}$

if and only if $\mathrm{C}_{t}>0$.

iii) is irreversible, if and only if the covariance function $C$ is non-constant. In this case the Boltzmann-Gibbs entropy

$S\left(\overline{\rho_{t}}\right):=-\left\langle\overline{\rho_{t}}, \ln \left(2 \pi \hbar \overline{\rho_{t}}\right)\right\rangle$

of $\overline{\rho_{t}}$ (if existent) is a strictly increasing function in time.

Proof. The first part of Statement 2.8 follows from Taylor expanding $\mathcal{N}$ in (2.23) with respect to $\hbar$

$\mathcal{N}=-D_{0,2} \partial_{p}^{2}-D_{1,1} \partial_{p} \partial_{q}-D_{2,0} \partial_{q}^{2}+\mathcal{O}\left(\hbar^{2} \partial^{4}\right)$,

with $D_{\mu, \nu}$ being defined in (2.39). In the limit $\hbar \searrow 0$ the expansion (2.52) reduces to the semielliptic differential operator $\mathcal{N}_{\mathrm{cl}}$. To obtain the right equality in (2.45) it is useful to note in addition the operator identity

$$
\left(\begin{array}{c}
\partial_{p} \\
\partial_{q}
\end{array}\right) \mathrm{e}^{-s \mathcal{L}}=\mathrm{e}^{-s \mathcal{L}} \mathrm{J}_{-s}^{\top}\left(\begin{array}{c}
\partial_{p} \\
\partial_{q}
\end{array}\right)
$$

which is a consequence of the chain rule and may be proved by applying both sides of (2.53) to a phase-space function. The second part of Statement 2.8 is an immediate consequence of the first part. To prove the third part, suppose that the Boltzmann-Gibbs entropy $S\left(\mathcal{T}_{t, \mathrm{cl}} \rho\right)$ exists. Since $\mathcal{T}_{t, \mathrm{cl}}$ is a semigroup it suffices to show $S\left(\mathcal{T}_{t, \mathrm{cl}} \rho\right)>S(\rho)$ if $t>0$ and $C$ is non-constant. To do so we conclude from $(2.49)$

$$
\begin{aligned}
S\left(\mathcal{T}_{t, \mathrm{cl}} \rho\right) & =-\int_{\mathbb{R} \times \mathbb{R}} P_{t, \mathrm{cl}}\left(\mathrm{d} p^{\prime} \mathrm{d} q^{\prime}\right)\left\langle\rho, \ln \left(2 \pi \hbar \mathcal{V}_{-p^{\prime},-q^{\prime}} \mathrm{e}^{t \mathcal{L}} \mathcal{T}_{t, \mathrm{cl}} \rho\right)\right\rangle \\
& >-\int_{\mathbb{R} \times \mathbb{R}} P_{t, \mathrm{cl}}\left(\mathrm{d} p^{\prime} \mathrm{d} q^{\prime}\right)\langle\rho, \ln (2 \pi \hbar \rho)\rangle \\
& =S(\rho),
\end{aligned}
$$

where $\mathcal{V}_{p^{\prime}, q^{\prime}}=\mathrm{e}^{p^{\prime} \partial_{p}+q^{\prime} \partial_{q}}$ denotes the translation operator by $\left(p^{\prime}, q^{\prime}\right)$. The strict inequality in (2.54) follows from the elementary inequality

$u-u \ln u<v-u \ln v, \quad u, v \geq 0, u \neq v$.

To see this we note that for $t>0$ and a non-constant $C$, the support of $P_{t, \mathrm{cl}}$ is at least a one-dimensional subspace of $\mathbb{R} \times \mathbb{R}$. The integrability of $\rho$ therefore forbids the equality

$\rho(p, q)=\int_{\mathbb{R} \times \mathbb{R}} P_{t, \mathrm{cl}}\left(\mathrm{d} p^{\prime \prime} \mathrm{d} q^{\prime \prime}\right) \rho\left(p^{\prime \prime}-p^{\prime}+p, q^{\prime \prime}-q^{\prime}+q\right)$ 
for $\rho \mathrm{d} p \mathrm{~d} q$-almost all $(p, q) \in \mathbb{R} \times \mathbb{R}$ and $P_{t, \mathrm{cl}}\left(\mathrm{d} p^{\prime} \mathrm{d} q^{\prime}\right)$-almost all $\left(p^{\prime}, q^{\prime}\right) \in$ $\mathbb{R} \times \mathbb{R}$.

Remark 2.9. The classical phase-space trajectories $\left(p_{t}, q_{t}\right)$ generated by the Hamiltonian $H+N_{t}$ realize a diffusion process on phase space. Its underlying stochastic differential equation coincides with Hamilton's equations of motion

$\partial_{t} p_{t}=-\left(\partial_{q}\left(H+N_{t}\right)\right)\left(p_{t}, q_{t}\right)$,

$\partial_{t} q_{t}=\left(\partial_{p}\left(H+N_{t}\right)\right)\left(p_{t}, q_{t}\right)$,

and (2.47) is the associated Fokker-Planck equation.

\subsection{Explicit results for averaged expectation values}

Now we return to the quantum-mechanical situation. In order to compute the averaged expectation value of an observable $a$, it is often advantageous to switch to the Heisenberg picture according to

$\overline{\left\langle w_{t}, a\right\rangle}=\left\langle\overline{w_{t}}, a\right\rangle=\left\langle\mathcal{T}_{t} w, a\right\rangle=\left\langle w, \mathcal{T}_{t}^{\dagger} a\right\rangle$.

The thus defined adjoint semigroup $\mathcal{T}_{t}^{\dagger}$ is obtained from $\mathcal{T}_{t}$ by simply reversing the sign of $\mathcal{L}$. In analogy to Statement 2.5 the adjoint semigroup has the representation

$$
\begin{aligned}
\mathcal{T}_{t}^{\dagger} & =\mathrm{e}^{t \mathcal{L}} \exp \left\{-\int_{0}^{t} \mathrm{~d} s \mathrm{e}^{-s \mathcal{L}} \mathcal{N} \mathrm{e}^{s \mathcal{L}}\right\} \\
& =\mathrm{e}^{t \mathcal{L}} \exp \left\{-\hbar^{-2} \int_{0}^{t} \mathrm{~d} s\left(C(0,0)-C\left(-\mathrm{i} \hbar \mathcal{K}_{-s}, \mathrm{i} \hbar \mathcal{X}_{-s}\right)\right)\right\}
\end{aligned}
$$

Statement 2.7 finds a similar translation. The expression (2.59b) serves as our starting point for obtaining some explicit results on the averaged dynamics. For example, the averaged Heisenberg picture of any observable which is a polynomial in $p$ and $q$ may be calculated from it. To this end we remark that there are only finitely many terms in the Taylor expansions of the right exponential in $(2.59 \mathrm{~b})$ and of the covariance function which contribute to the result. Examples for the cases where the deterministic part of the Hamiltonian is that of a free particle or that of a particle subjected to a constant magnetic field in two space dimensions have been reported in Fischer et al. (1994). Here we only recall the free-particle case.

Example 2.10. Let $H=p^{2} /(2 m)$. Then

$$
\begin{aligned}
& \mathcal{T}_{t}^{\dagger} p=p, \\
& \mathcal{T}_{t}^{\dagger} q=q+t p / m, \\
& \mathcal{T}_{t}^{\dagger} p^{2}=p^{2}+2 t D_{0,2}, \\
& \mathcal{T}_{t}^{\dagger} p q=p(q+t p / m)+t D_{1,1}+t^{2} D_{0,2} / m, \\
& \mathcal{T}_{t}^{\dagger} q^{2}=(q+t p / m)^{2}+2 t D_{2,0}+t^{2} D_{1,1} / m+\frac{2}{3} t^{3} D_{0,2} / m^{2}, \\
& \mathcal{T}_{t}^{\dagger} q^{4}=(q+t p / m)^{4}+12\left\{(q+t p / m)^{2} d_{2}(t)+2 \hbar^{2} d_{4}(t)+\left(d_{2}(t)\right)^{2}\right\} .
\end{aligned}
$$


The coefficients $D_{\mu, \nu}$ were defined in (2.39) and we have introduced the polynomials $d_{\mu}(t):=\sum_{\nu=0}^{\mu} \frac{D_{\mu-\nu, \nu}}{(\nu+1) m^{\nu}} t^{\nu+1}$ of maximal degree $\mu+1$. As a consequence, the long-time spreading of states under the averaged dynamics is diffusive in momentum and superballistic in position

$$
\begin{aligned}
& \lim _{t \rightarrow \infty} \frac{1}{t}\left(\left\langle\overline{w_{t}}, p^{2}\right\rangle-\left\langle\overline{w_{t}}, p\right\rangle^{2}\right)=2 D_{0,2}, \\
& \lim _{t \rightarrow \infty} \frac{1}{t^{3}}\left(\left\langle\overline{w_{t}}, q^{2}\right\rangle-\left\langle\overline{w_{t}}, q\right\rangle^{2}\right)=\frac{2 D_{0,2}}{3 m^{2}} .
\end{aligned}
$$

Example 2.10 illustrates important features valid for general quadratic $H$ : Observables which are linear in $p$ and $q$ are not affected by the white noise $N_{t}$. Moreover, noise-induced terms in averaged expectation values of quadratic observables are independent of the initial state. Assuming that the deterministic part $H$ of the Hamiltonian and the covariance function $C$ are $\hbar$ independent, noise-induced effects affected by quantum fluctuations occur only for observables of at least fourth order in $p$ or $q$. However, very special situations are needed for quantum effects to show up in the leading term for long times. For example, taking the observables $p^{n}$ or $q^{n}, 4 \leq n$ integer, one must require the phase-space trajectories of $H$ to grow exponentially in time. These results generalize some earlier ones in Jayannavar and Kumar (1982), Heinrichs (1983b), Ghirardi et al. (1986), Heinrichs (1992) and Jayannavar (1993).

Due to the time dependence of the random part $N_{t}$ of the Hamiltonian, one does not expect energy conservation for white-noise systems. The observable $\mathcal{T}_{t}^{\dagger} H$ can be interpreted as the mean energy of the system at time $t$. For the special case considered in Example 2.10, the mean energy grows linearly in time, see Eq. (2.60c). This is also true under more general circumstances.

Statement 2.11. For an at most quadratic deterministic part $H$, the mean energy changes at a constant rate:

$\mathcal{T}_{t}^{\dagger} H=H+t\left(D_{0,2}\left(\partial_{p}^{2} H\right)+D_{1,1}\left(\partial_{p} \partial_{q} H\right)+D_{2,0}\left(\partial_{q}^{2} H\right)\right)$.

The rate does not allow for a dependence on the initial state.

Proof. The claim follows from (2.59a), from $\mathrm{e}^{s \mathcal{L}} H=H$, from

$-\mathcal{N} H=D_{0,2}\left(\partial_{p}^{2} H\right)+D_{1,1}\left(\partial_{p} \partial_{q} H\right)+D_{2,0}\left(\partial_{q}^{2} H\right)$

and from $\mathcal{N}^{2} H=0$. Note also that the right-hand side in (2.63) does not depend on $p$ and $q$.

Unless $H$ is unbounded from below, such as $H=\left(p^{2}-D q^{2}\right) /(2 m)$ for $D>$ $D_{0,2} / D_{2,0}$, the mean energy increases to infinity in the course of time. To compensate for this effect and, if possible, to allow for an eventual approach to a stationary equilibrium state, dissipation has to be incorporated. This will be done in the next section by coupling the white-noise system to a heat bath. 


\section{White noise and dissipation}

This section is devoted to the interplay of white noise and dissipation. Such an investigation is physically sensible, because the additive white noise in Model 2.1 is in general not due to thermal fluctuations. We will show that the incorporation of dissipation causes the mean energy of the white-noise system to saturate for long times. Topics of this kind were already addressed to in Heinrichs (1983a), where dissipation is generated through an ad-hoc time dependence of the deterministic part of the Hamiltonian. In contrast, we introduce dissipation by letting the white-noise system interact with a microscopic model of a heat bath - a more physical strategy which is commonly accepted today (Magalinskiǔ, 1959; Feynman and Vernon, 1963; Ford et al., 1965; Ullersma, 1966; Arai, 1981; Caldeira and Leggett, 1983; Haake and Reibold, 1985; Ford et al., 1988a; Grabert et al., 1988; Sogo and Fujimoto, 1990; Weiss, 1993; Efimov and von Waldenfels, 1994; Jakšić and Pillet, 1997a).

The programme of this section will be accomplished in four steps: First, the white-noise system is bilinearly coupled to a heat bath of independent harmonic oscillators. The averaged dynamics of the total system, that is, of the white-noise system plus heat bath, is then studied with the methods of the preceding section. Second, the bath variables are eliminated assuming that the bath is in thermal equilibrium. Third, the thus obtained reduced averaged dynamics is considered in the macroscopic limit of the heat bath. Finally, the long-time limit is analyzed. Statement 3.12 summarizes the main result.

\subsection{The averaged dynamics of the total system}

For notational simplicity, we will no longer allow for a momentum dependence of the white noise and we will restrict the deterministic part of the Hamiltonian to that of a harmonic oscillator. Note also that in this section we are using the Weyl-Wigner-Moyal representation for a quantum-mechanical system with several Cartesian degrees of freedom.

Model 3.1. The Hamiltonian of the total system is defined by the WeylWigner symbol

$H^{(n)}+N_{t}$

on phase space $\mathbb{R}^{n+1} \times \mathbb{R}^{n+1}$. The zeroth components $(p, q)$ of $\mathbf{p}:=$ $\left(p, p_{1}, \ldots, p_{n}\right) \in \mathbb{R}^{n+1}$ and $\mathbf{q}:=\left(q, q_{1}, \ldots, q_{n}\right) \in \mathbb{R}^{n+1}$ make up the phasespace coordinates of the white-noise system, the following components those of the heat bath. The deterministic part

$H^{(n)}(\mathbf{p}, \mathbf{q}):=p^{2} /(2 m)+\left(m \omega^{2} / 2\right) q^{2}+\sum_{j=1}^{n}\left\{p_{j}^{2} /\left(2 m_{j}\right)+\left(m_{j} \omega_{j}^{2} / 2\right)\left(q_{j}-q\right)^{2}\right\}$ 
of (3.1) couples a harmonic oscillator of mass $m>0$ and frequency $\omega \geq 0$ bilinearly to a heat bath of $n$ independent harmonic oscillators with masses $m_{j}>0, j=1, \ldots, n$, and frequencies $0<\omega_{1}<\omega_{2}<\ldots<\omega_{n}$. The random part

$N_{t}(\mathbf{p}, \mathbf{q}) \equiv N_{t}(q)$

defines a time-dependent local potential on the phase space of the white-noise system only. It is a Gaussian random field with zero mean and covariance function

$\overline{N_{t}(q) N_{t^{\prime}}\left(q^{\prime}\right)}=\delta\left(t-t^{\prime}\right) C\left(q-q^{\prime}\right)$.

Introducing the diagonal $(n+1) \times(n+1)$-mass matrix

$\mathbf{M}:=\operatorname{diag}\left(m, m_{1}, m_{2}, \ldots, m_{n}\right)>0$

and the $(n+1) \times(n+1)$-matrix of squared frequencies

$\Omega^{2}:=\left(\begin{array}{ccccc}\omega^{2}+\sum_{j=1}^{n} \kappa_{j}^{2} \omega_{j}^{2} & -\kappa_{1} \omega_{1}^{2} & \ldots & -\kappa_{n} \omega_{n}^{2} \\ -\kappa_{1} \omega_{1}^{2} & \omega_{1}^{2} & & 0 \\ \cdot & & \cdot & & \\ \cdot & & & . & \\ \cdot & & & \omega_{n}^{2}\end{array}\right) \geq 0$

where $\kappa_{j}:=\sqrt{m_{j} / m}, j=1, \ldots, n$, one can rewrite (3.2) as a non-negative quadratic form

$H^{(n)}(\mathbf{p}, \mathbf{q})=\frac{1}{2} \mathbf{p} \cdot \mathrm{M}^{-1} \mathbf{p}+\frac{1}{2} \mathbf{q} \cdot \mathrm{M}^{1 / 2} \Omega^{2} \mathrm{M}^{1 / 2} \mathbf{q}$.

This model for the heat bath is referred to in the literature as the independent oscillator model (Ford et al., 1988a,b) or Caldeira-Leggett model (Weiss, 1993).

Following the strategy of the preceding section we define the averaged dynamics of the total system as

$\mathcal{T}_{t}^{(n)}:=\lim _{\tau \searrow 0} \overline{\exp \left\{-\int_{0}^{t} \mathrm{~d} s\left[H^{(n)}+N_{s}^{(\tau)}, \bullet\right]\right\}}$,

where $N_{t}^{(\tau)}$ is a Gaussian coloured noise which converges to the Gaussian white noise $N_{t}$ of (3.3) in the limit of vanishing correlation time $\tau \searrow 0$, confer Subsection 2.1.

The validity of the following statement depends only on the quadratic form (3.7) of $H^{(n)}$. The specific forms of the matrices $\mathrm{M}$ and $\Omega^{2}$ are irrelevant. 
Statement 3.2. The averaged dynamics $\mathcal{T}_{t}^{(n)}$ is a semigroup and admits the representation

$\mathcal{T}_{t}^{(n)}=\mathrm{e}^{-t \mathcal{L}^{(n)}} \exp \left\{-\hbar^{-2} \int_{0}^{t} \mathrm{~d} s\left(C(0)-C\left(\mathrm{i} \hbar \mathcal{X}_{s}^{(n)}\right)\right)\right\}$.

Here we have introduced the differential operator

$\mathcal{X}_{s}^{(n)}:=\left(\mathrm{M}^{-1 / 2} \cos (\Omega s) \mathrm{M}^{1 / 2} \partial_{\mathbf{p}}-\mathrm{M}^{-1 / 2} \frac{\sin (\Omega s)}{\Omega} \mathrm{M}^{-1 / 2} \partial_{\mathbf{q}}\right)_{0}$

as the zeroth component of the vector on the right-hand side of (3.10). The superscript $n$ accounts for the fact that the Liouvillian which generates the reversible dynamics of the deterministic part of the total Hamiltonian is $\mathcal{L}^{(n)}:=\left[H^{(n)}, \bullet\right]$.

Proof. The semigroup property is established as in the proof of Statement 2.2. To arrive at the representation (3.9) we remark that the classical phasespace trajectories generated by $\mathcal{L}^{(n)}$ are given by

$\mathrm{e}^{t \mathcal{L}^{(n)}} \mathbf{p}=\mathrm{M}^{1 / 2} \cos (\Omega t) \mathrm{M}^{-1 / 2} \mathbf{p}-\mathrm{M}^{1 / 2} \Omega \sin (\Omega t) \mathrm{M}^{1 / 2} \mathbf{q}$
$\mathrm{e}^{t \mathcal{L}^{(n)}} \mathbf{q}=\mathrm{M}^{-1 / 2} \frac{\sin (\Omega t)}{\Omega} \mathrm{M}^{-1 / 2} \mathbf{p}+\mathrm{M}^{-1 / 2} \cos (\Omega t) \mathrm{M}^{1 / 2} \mathbf{q}$

and that the differential operators $\mathcal{K}_{s}$ and $\mathcal{X}_{s}$ introduced in Statement 2.5 are to be replaced in the multi-dimensional case by vector-valued differential operators whose $j$-th components, $j \in\{0,1, \ldots, n\}$, read

$\left(\mathcal{K}_{s}\right)_{j}:=\left(\partial_{q_{j}} \mathrm{e}^{-s \mathcal{L}} \mathbf{p}\right) \cdot \partial_{\mathbf{p}}+\left(\partial_{q_{j}} \mathrm{e}^{-s \mathcal{L}} \mathbf{q}\right) \cdot \partial_{\mathbf{q}}$,

$\left(\mathcal{X}_{s}\right)_{j}:=\left(\partial_{p_{j}} \mathrm{e}^{-s \mathcal{L}} \mathbf{p}\right) \cdot \partial_{\mathbf{p}}+\left(\partial_{p_{j}} \mathrm{e}^{-s \mathcal{L}} \mathbf{q}\right) \cdot \partial_{\mathbf{q}}$.

In this notation Definition (3.10) appears as $\mathcal{X}_{s}^{(n)}=\left(\mathcal{X}_{s}\right)_{0}$.

To describe the averaged dynamics of observables we introduce the adjoint $\left(\mathcal{T}_{t}^{(n)}\right)^{\dagger}$ of the semigroup $\mathcal{T}_{t}^{(n)}$ with respect to the standard scalar product

$\langle f, g\rangle:=\int_{\mathbb{R}^{n+1} \times \mathbb{R}^{n+1}} \mathrm{~d}^{n+1} p \mathrm{~d}^{n+1} q f^{*}(\mathbf{p}, \mathbf{q}) g(\mathbf{p}, \mathbf{q})$

for square-integrable functions $f, g$ on phase space $\mathbb{R}^{n+1} \times \mathbb{R}^{n+1}$.

Since we are mostly interested in the behaviour of the mean energy, it suffices to concentrate on the averaged dynamics of the system observables $p^{2}$ and $q^{2}$, and thus on the spreading of an averaged state of the white-noise system. Again, explicit results on the averaged dynamics of polynomial observables may be obtained from a Taylor expansion of the adjoint semigroup. 
Statement 3.3.

$$
\begin{aligned}
& \left(\mathcal{T}_{t}^{(n)}\right)^{\dagger} p^{2}=\left(\mathrm{e}^{t \mathcal{L}^{(n)}} p\right)^{2}+\left(-\partial_{q}^{2} C\right)(0) \int_{0}^{t} \mathrm{~d} s\left\{\left(\mathrm{M}^{-1 / 2} \cos (\Omega s) \mathrm{M}^{1 / 2}\right)_{00}\right\}^{2} \\
& \left(\mathcal{T}_{t}^{(n)}\right)^{\dagger} q^{2}=\left(\mathrm{e}^{t \mathcal{L}^{(n)}} q\right)^{2}+\left(-\partial_{q}^{2} C\right)(0) \int_{0}^{t} \mathrm{~d} s\left\{\left(\mathrm{M}^{-1 / 2} \frac{\sin (\Omega s)}{\Omega} \mathrm{M}^{-1 / 2}\right)_{00}\right\}^{2}
\end{aligned}
$$

\subsection{Elimination of the bath variables and the quantum Langevin equation}

The second step of the programme outlined at the beginning of this section is to eliminate the degrees of freedom of the heat bath assuming that the bath is in a thermal equilibrium state. This is achieved with the help of the partial expectation value

$$
\begin{aligned}
\left\langle a_{n}\right\rangle_{\beta, n}(p, q) & :=\int_{\mathbb{R}^{n} \times \mathbb{R}^{n}} \mathrm{~d} p_{1} \mathrm{~d} q_{1} \ldots \mathrm{d} p_{n} \mathrm{~d} q_{n} a_{n}(\mathbf{p}, \mathbf{q}) \\
\times & \left(\prod_{j=1}^{n} \frac{\omega_{j} \beta_{\mathrm{eff}}\left(\hbar \omega_{j}\right)}{2 \pi} \exp \left\{-\beta_{\mathrm{eff}}\left(\hbar \omega_{j}\right)\left(p_{j}^{2} /\left(2 m_{j}\right)+\left(m_{j} \omega_{j}^{2} / 2\right) q_{j}^{2}\right)\right\}\right)
\end{aligned}
$$

of an observable $a_{n}$ of the total system. Consequently, we do not allow for initial correlations between the white-noise system and the heat bath. The phase-space function in the second line of (3.15) is the Wigner density of the canonical equilibrium state of the $n$ bath oscillators at inverse temperature $\beta<\infty$. Note that quantum fluctuations of the heat bath are fully taken into account through effectively increased temperatures, defined by the function

$$
E \mapsto \beta_{\mathrm{eff}}(E):=\frac{2}{E} \tanh \left(\frac{\beta E}{2}\right) .
$$

Starting from the Heisenberg equations of motion for the $n+1$ components of position and momentum of the total system, one deduces that $\mathrm{e}^{t \mathcal{L}^{(n)}} q$ satisfies a linear quantum Langevin equation (Ford et al., 1965; Benguria and Kac, 1981; Maassen, 1984; Ford et al., 1987, 1988a; Gardiner, 1991; Jakšić and Pillet, 1997a) - derivatives with respect to time are symbolized by superposed dots-

$\ddot{x}(t)+m^{-1} \int_{0}^{t} \mathrm{~d} s \gamma_{n}(t-s) \dot{x}(s)+\omega^{2} x(t)+\gamma_{n}(t) x(0) / m=F_{n}(t) / m$

with the friction kernel

$\gamma_{n}(t):=\sum_{j=1}^{n} m_{j} \omega_{j}^{2} \cos \left(\omega_{j} t\right)$ 
and the driving force

$F_{n}(t):=\sum_{j=1}^{n}\left\{m_{j} \omega_{j}^{2} q_{j} \cos \left(\omega_{j} t\right)+\omega_{j} p_{j} \sin \left(\omega_{j} t\right)\right\}$.

The latter behaves with respect to the partial expectation value $\langle\cdot\rangle_{\beta, n}$ as a (classical) stationary Gaussian stochastic process with zero mean and covariance function

$\left\langle F_{n}(t) F_{n}\left(t^{\prime}\right)\right\rangle_{\beta, n}=\sum_{j=1}^{n} \frac{m_{j} \omega_{j}^{2}}{\beta_{\mathrm{eff}}\left(\hbar \omega_{j}\right)} \cos \left(\omega_{j}\left(t-t^{\prime}\right)\right)=: \Phi_{\beta, n}\left(t-t^{\prime}\right)$.

Observe that the linear quantum Langevin equation (3.17) in the Weyl-Wigner-Moyal representation differs from the corresponding classical Langevin equation (Anglin and Habib, 1996; Jakšić and Pillet, 1997b) only by the effective temperatures in the covariance function of the driving force.

Thus we have shown

Statement 3.4. The classical phase-space trajectories generated by $H^{(n)}$ can be written as

$\mathrm{e}^{t \mathcal{L}^{(n)}} p=m \partial_{t} \mathrm{e}^{t \mathcal{L}^{(n)}} q$,

$\mathrm{e}^{t \mathcal{L}^{(n)}} q=(p / m) G_{n}(t)+q \dot{G}_{n}(t)+m^{-1} \int_{0}^{t} \mathrm{~d} s G_{n}(t-s) F_{n}(s)$.

Here

$G_{n}(t):=\int_{\mathbb{R}+\mathrm{i} \varepsilon} \frac{\mathrm{d} z}{2 \pi} \frac{\mathrm{e}^{-\mathrm{i} z t}}{\omega^{2}-z^{2}-\mathrm{i} z \hat{\gamma}_{n}(z) / m}, \quad t \in \mathbb{R}, \varepsilon>0$,

is the retarded Green function (or causal response function) of the quantum Langevin equation (3.17) and

$\hat{\gamma}_{n}(z):=\int_{0}^{\infty} \mathrm{d} t \mathrm{e}^{\mathrm{i} z t} \gamma_{n}(t)=\frac{\mathrm{i}}{2} \sum_{j=1}^{n} m_{j} \omega_{j}^{2}\left(\frac{1}{z-\omega_{j}}+\frac{1}{z+\omega_{j}}\right)$

is the Laplace transform of the friction kernel (3.18) defined for $z \in \mathbb{C}$, $\operatorname{Im} z>0$.

Remarks 3.5. i) One deduces for $\operatorname{Im} z>0$ from the explicit expression (3.23) that

$\operatorname{Im}\left\{\frac{\omega^{2}-z^{2}}{z}\right\}<0<\operatorname{Im}\left\{\mathrm{i} \hat{\gamma}_{n}(z)\right\}$,

Hence, the denominator of the integrand in (3.22) has no zeros in the complex upper half-plane. Moreover, the integrand of (3.22) is analytic in this half-plane, and therefore the definition of the Green function $G_{n}$ does not depend on $\varepsilon$. 
ii) Note that $\lim _{t \searrow 0} G_{n}(t)=0, \lim _{t \searrow 0} \dot{G}_{n}(t)=1$ and $\lim _{t \searrow 0} \ddot{G}_{n}(t)=0$.

iii ) The Laplace transforms of $G_{n}$ and its derivatives read for $z \in \mathbb{C}, \operatorname{Im} z>$ 0 ,

$$
\begin{aligned}
& \hat{G}_{n}(z):=\int_{0}^{\infty} \mathrm{d} t \mathrm{e}^{\mathrm{i} z t} G_{n}(t)=\frac{1}{\omega^{2}-z^{2}-\mathrm{i} z \hat{\gamma}_{n}(z) / m}, \\
& \hat{\dot{G}}_{n}(z)=-\mathrm{i} z \hat{G}_{n}(z), \\
& \hat{\tilde{G}}_{n}(z)=-z^{2} \hat{G}_{n}(z)-1 .
\end{aligned}
$$

Basically, Statement 3.4 reduces the analysis of the first summands of $\left(\mathcal{T}_{t}^{(n)}\right)^{\dagger} p^{2}$ and $\left(\mathcal{T}_{t}^{(n)}\right)^{\dagger} q^{2}$, as given by (3.14), to an analysis of the Green function $G_{n}$. The next statement does the same for the second summands in (3.14).

Statement 3.6. Let $\mathcal{S}_{\delta}:=\{z \in \mathbb{C}:|\operatorname{Im} z|<\delta\}$ be a strip of width $2 \delta>0$ centred around the real axis in the complex plane and let $f: \mathcal{S}_{\delta} \longrightarrow \mathbb{C}$ be a bounded analytic function on $\mathcal{S}_{\delta}$ with reflection symmetry $f(z)=f(-z)$ for all $z \in \mathcal{S}_{\delta}$. Then, for any $0<\varepsilon<\delta$, the 00 -matrix element $(f(\Omega))_{00}$, where $\Omega$ stands for the positive square root of the $(n+1) \times(n+1)$-matrix of squared frequencies (3.6), may be expressed in terms of a principal-value integral

$$
(f(\Omega))_{00}=\frac{1}{\pi \mathrm{i}} \mathrm{PV} \int_{\mathbb{R}+\mathrm{i} \varepsilon} \mathrm{d} z z f(z) \hat{G}_{n}(z) .
$$

Proof. We closely follow Ullersma (1966) and Ford et al. (1988b). Using the spectral representation $\Omega=\mathrm{U} \operatorname{diag}\left(\Omega_{0}, \ldots, \Omega_{n}\right) \mathrm{U}^{\mathrm{t}}$, where $\mathrm{U}$ is an orthogonal $(n+1) \times(n+1)$-Matrix and $0 \leq \Omega_{0} \leq \Omega_{1} \leq \ldots \leq \Omega_{n}$ are the eigenvalues of $\Omega$, the left-hand side of (3.26) is rewritten as

$$
(f(\Omega))_{00}=\sum_{k=0}^{n}\left(\mathrm{U}_{0 k}\right)^{2} f\left(\Omega_{k}\right) .
$$

The proof of the following claim is contained in Ford et al. (1988b).

The function

$\xi \mapsto \alpha(\xi):=\hat{G}_{n}(\sqrt{\xi})=\frac{1}{\omega^{2}-\xi-\mathrm{i} \sqrt{\xi} \hat{\gamma}_{n}(\sqrt{\xi}) / m}$

extends to an analytic function on $\mathbb{C} \backslash\left\{\Omega_{0}^{2}, \ldots, \Omega_{n}^{2}\right\}$. Moreover, $\Omega_{j} \neq \Omega_{k}$ for $j \neq k$, and $\alpha$ has a simple pole at $\xi=\Omega_{k}^{2}$ with residue $\operatorname{Res}_{\Omega_{k}^{2}}\{\alpha\}=-\left(\mathrm{U}_{0 k}\right)^{2}$ for $k=0, \ldots, n$.

Due to the symmetry $f(z)=f(-z)$ there exists an analytic function $g$ on $\mathcal{S}_{\delta}^{2}:=\left\{\xi \in \mathbb{C}: \xi=z^{2}, z \in \mathcal{S}_{\delta}\right\}$ such that $g\left(z^{2}\right)=f(z)$. Hence, the 

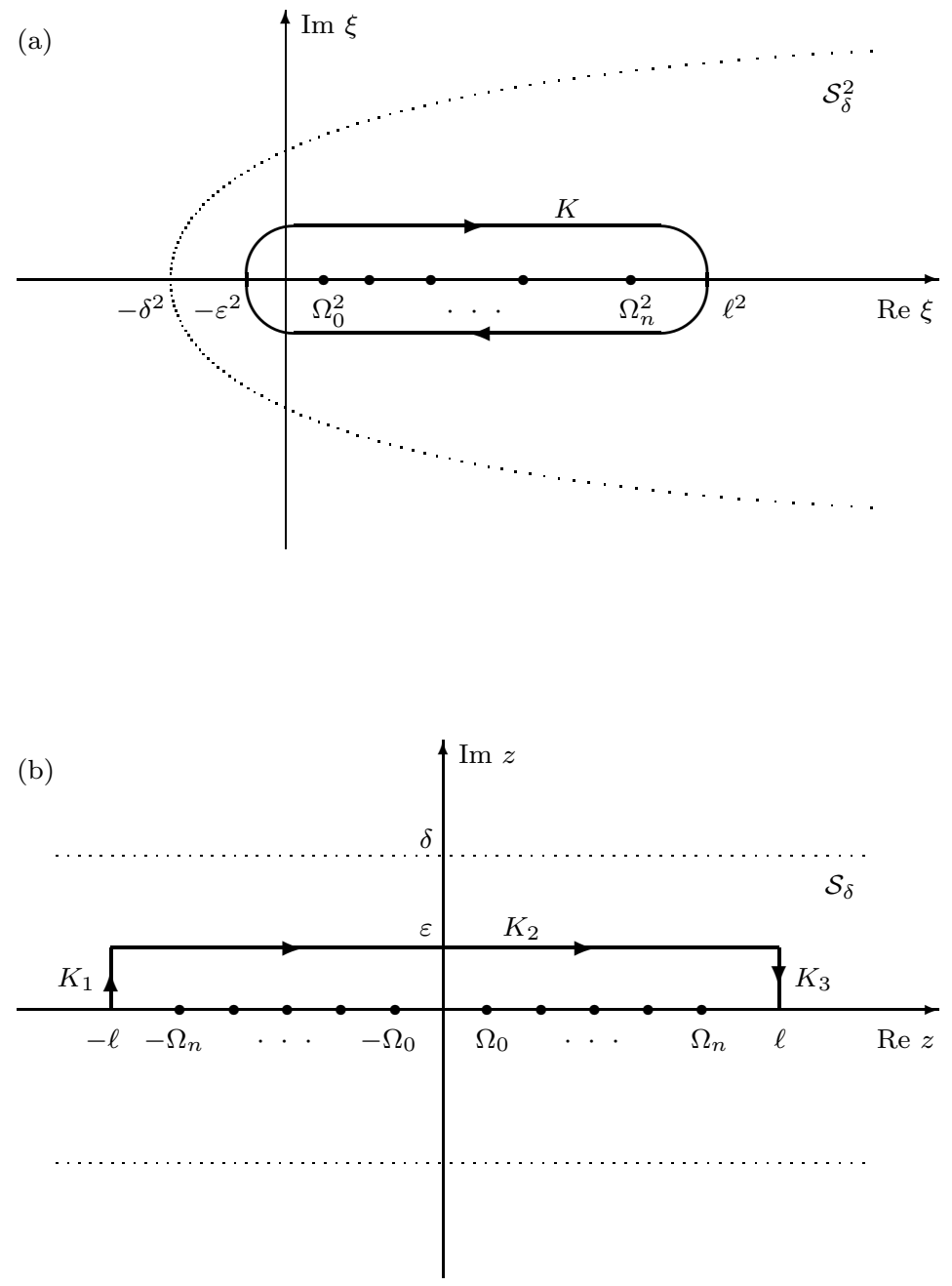

Fig. 2. The poles of the integrand and the integration contour for (a) the integral (3.29) and (b) the integral (3.30). 
above claim and (3.27) lead in combination with the residue theorem to the representation

$$
(f(\Omega))_{00}=\frac{1}{2 \pi \mathrm{i}} \int_{K} \mathrm{~d} \xi g(\xi) \alpha(\xi) .
$$

As is depicted in Figure 2(a), the integration contour $K$ encloses clockwise all poles of $\alpha$. It intersects the real axis at $\xi=-\varepsilon^{2}$ and at $\xi=\ell^{2}$, where $0<\varepsilon<\delta$ and $\ell>\Omega_{n}$. Next we introduce $z:=\sqrt{\xi}$ as the square root of $\xi$ with branch cut along the positive real half-axis and perform a change-of-variables in the integral (3.29)

$$
(f(\Omega))_{00}=\frac{1}{\pi \mathrm{i}} \int_{K_{1}+K_{2}+K_{3}} \mathrm{~d} z z f(z) \alpha\left(z^{2}\right),
$$

see Figure 2(b). In the limit $\ell \rightarrow \infty$ the contributions of the vertical line segments $K_{1}$ and $K_{3}$ to the integral (3.30) vanish due to the boundedness of $f$, whereas the contribution of the horizontal line segment $K_{2}$ converges to the principal-value integral in (3.26).

\subsection{The macroscopic limit and the reduced averaged dy- namics}

In order to enable the white-noise system to dissipate energy into the heat bath, it is necessary to take the macroscopic limit of the heat bath such that a continuum of frequencies arises. More precisely, guided by the relation

$\gamma_{n}(t)=\int_{\mathbb{R}} \mathrm{d} \nu J_{n}(\nu) \mathrm{e}^{\mathrm{i} \nu t}$,

where $J_{n}(\nu):=\frac{1}{2} \sum_{j=1}^{n} m_{j} \omega_{j}^{2}\left[\delta\left(\nu-\omega_{j}\right)+\delta\left(\nu+\omega_{j}\right)\right]$, we require that with the number $n$ of bath oscillators going to infinity, the limit of the friction kernel $\gamma(t):=\lim _{n \rightarrow \infty} \gamma_{n}(t)$ exists and admits a representation as the Fourier transform of the so-called spectral density $J$ of the macroscopic heat bath

$\gamma(t)=\int_{\mathbb{R}} \mathrm{d} \nu J(\nu) \mathrm{e}^{\mathrm{i} \nu t}$.

The spectral density describes the strength of the coupling between the system and the heat bath. In what follows, it is supposed to satisfy

Assumptions 3.7. $\quad$ i) $J$ is continuous, even and strictly positive, $J(\nu)=$ $J(-\nu)>0$ for all $\nu \in \mathbb{R}$.

ii) There are two constants $0<\psi<\pi / 2$ and $\delta>0$ such that $J$ admits an analytic continuation to the subset $\mathcal{H}_{\psi, \delta}^{-}:=\{z \in \mathbb{C}: \operatorname{Im} z<$ 0 and $\arg (z+\mathrm{i} \delta) \notin[-\pi+\psi,-\psi]\}$ of the complex lower half-plane, see Figure 3. 


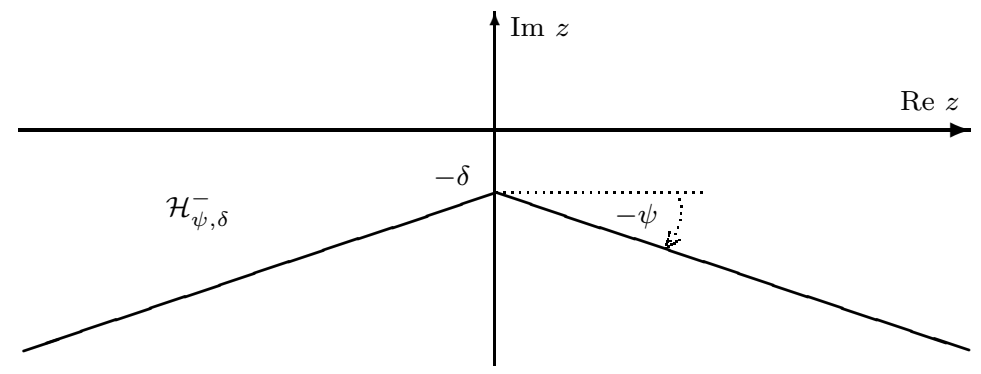

Fig. 3. The subset $\mathcal{H}_{\psi, \delta}^{-}:=\{z \in \mathbb{C}: \operatorname{Im} z<0, \arg (z+\mathrm{i} \delta) \notin[-\pi+\psi,-\psi]\}$ of the complex plane.

iii) $J$ has the following decay properties:

$\sup _{z \in \mathcal{H}_{\psi, \delta}^{-}}\left|z^{2} J(z)\right|<\infty$ and the limit $L:=\lim _{|\nu| \rightarrow \infty} \nu^{2} J(\nu)$, taken along the real axis, exists and is approached sufficiently fast,

$$
\lim _{|\nu| \rightarrow \infty}\left\{|\nu|^{r}\left(\nu^{2} J(\nu)-L\right)\right\}=0 \quad \text { for some } r>0 .
$$

Note that Assumptions 3.7.i) and 3.7.iii) imply the integrability of $J$ over $\mathbb{R}$.

Assumptions 3.7 are obviously satisfied for the Gaussian spectral density $J(\nu)=J_{0} \exp \left\{-\nu^{2} /\left(2 \omega_{0}^{2}\right)\right\}, J_{0}, \omega_{0}>0$. Another admissible choice for $J$ is the Cauchy-Lorentz spectral density $J(\nu)=J_{0} \omega_{0}^{2} /\left(\omega_{0}^{2}+\nu^{2}\right)$ of the so-called Drude model. Both densities approximate Ohmic damping, which is defined by $J(\nu)=J_{0}$ for all $\nu$, the better the larger $\omega_{0}$ is. Ohmic damping itself is excluded by the decay assumption 3.7.iii). It leads for $\hbar \neq 0$ to some unphysical ultraviolet divergencies, see for example (3.34) and (3.53a) below. The reader should be aware that the recent attempt of Ford and O'Connell (1996) to interpret some of these divergencies in a naïve distributional sense has failed: the proposed distributional derivative of the hyperbolic cotangent makes only sense for test functions which vanish at the origin. But this implies that the distributional derivative does not differ from the classical one.

For later purpose we state that the covariance function (3.20) of the driving force $F_{n}$ in the quantum Langevin equation evaluates in the macroscopic limit to

$\Phi_{\beta}(t):=\lim _{n \rightarrow \infty} \Phi_{\beta, n}(t)=(\hbar / 2) \int_{\mathbb{R}} \mathrm{d} \nu J(\nu) \nu \operatorname{coth}(\beta \hbar \nu / 2) \mathrm{e}^{\mathrm{i} \nu t}$.

Accordingly, its Fourier transform differs from that of the friction kernel simply by the factor of $1 / \beta_{\text {eff }}(\hbar \nu)$, a fact which reflects the fluctuation-dissipation 
theorem (Kubo et al., 1991). Furthermore we use the notation

$G(t):=\lim _{n \rightarrow \infty} G_{n}(t)$

for the macroscopic limit of the Green function of the quantum Langevin equation. It does not only depend on the spectral density $J$ of the heat bath, but also on the mass $m$ and the frequency $\omega$ of the system Hamiltonian.

After these preparations and recalling the partial expectation value (3.15) with respect to the canonical equilibrium state at inverse temperature $\beta$ of the heat bath, the reduced averaged dynamics of system observables on phase space $\mathbb{R} \times \mathbb{R}$ is defined as

$$
\left(\mathcal{R}_{t}^{(\beta, J)}\right)^{\dagger}:=\lim _{n \rightarrow \infty}\left\langle\left(\mathcal{T}_{t}^{(n)}\right)^{\dagger} \cdot\right\rangle_{\beta, n},
$$

confer (3.8) and (3.15). Its adjoint $\mathcal{R}_{t}^{(\beta, J)}$ with respect to the standard scalar product for square-integrable functions on phase space $\mathbb{R} \times \mathbb{R}$ describes the reduced averaged dynamics for the states of the white-noise system.

The starting point for deriving the long-time spreading of states under this dynamics is

\section{Statement 3.8.}

$$
\begin{aligned}
\left(\mathcal{R}_{t}^{(\beta, J)}\right)^{\dagger} p^{2}=(p \dot{G}(t)+m q \ddot{G}(t))^{2} & +\int_{0}^{t} \mathrm{~d} s \int_{0}^{t} \mathrm{~d} s^{\prime} \dot{G}(s) \Phi_{\beta}\left(s-s^{\prime}\right) \dot{G}\left(s^{\prime}\right) \\
& +\left(-\partial_{q}^{2} C\right)(0) \int_{0}^{t} \mathrm{~d} s(\dot{G}(s))^{2} \\
\left(\mathcal{R}_{t}^{(\beta, J)}\right)^{\dagger} q^{2}=\left(\frac{p}{m} G(t)+q \dot{G}(t)\right)^{2} & +m^{-2} \int_{0}^{t} \mathrm{~d} s \int_{0}^{t} \mathrm{~d} s^{\prime} G(s) \Phi_{\beta}\left(s-s^{\prime}\right) G\left(s^{\prime}\right) \\
& +m^{-2}\left(-\partial_{q}^{2} C\right)(0) \int_{0}^{t} \mathrm{~d} s(G(s))^{2} .
\end{aligned}
$$

Proof. The terms in the first line of (3.37a) and (3.37b), respectively, follow from taking the partial expectation value (3.15) of the first term in (3.14a) and $(3.14 \mathrm{~b})$, respectively. It is evaluated in the macroscopic limit by using Statement 3.4, as well as (3.20), (3.34) and (3.35). The term in the second line of $(3.37 \mathrm{~b})$ emerges in the macroscopic limit from the second term in (3.14b) due to

$$
\left(\frac{\sin (\Omega s)}{\Omega}\right)_{00}=-\int_{\mathbb{R}+\mathrm{i} \varepsilon} \frac{\mathrm{d} z}{2 \pi}\left(\mathrm{e}^{\mathrm{i} z s}-\mathrm{e}^{-\mathrm{i} z s}\right) \hat{G}_{n}(z)=G_{n}(s), \quad s \geq 0 .
$$

Here we have applied Statement 3.6 and used the causality condition

$G_{n}(s)=0$ for all $s \leq 0$,

which is a consequence of Cauchy's integral theorem for analytic functions and Definition (3.22). As to the term in the second line of (3.37a), we simply refer to $(3.38)$ and to $\cos (\Omega s)=\partial_{s} \sin (\Omega s) / \Omega$. 


\subsection{The long-time limit}

The dissipative character of the reduced averaged dynamics will become obvious from the long-time behaviour of the Green function $G$ and of the covariance function $\Phi_{\beta}$. In fact, under our Assumptions 3.7 these functions exhibit an exponential relaxation.

Statement 3.9. There is a decay constant $\eta>0$ such that the long-time behaviour of the macroscopic Green function (3.35) and of its derivatives is given by

$G(t)= \begin{cases}(\pi J(0))^{-1}+\mathcal{O}\left(\mathrm{e}^{-\eta t}\right), & \text { if } \omega=0, \\ \mathcal{O}\left(\mathrm{e}^{-\eta t}\right), & \text { if } \omega>0,\end{cases}$

$\dot{G}(t)=\mathcal{O}\left(\mathrm{e}^{-\eta t}\right)$,

$\ddot{G}(t)=\mathcal{O}\left(\mathrm{e}^{-\eta t}\right)$.

The covariance function $\Phi_{\beta}$ from (3.34) is Lebesgue integrable over $\mathbb{R}$. It decays exponentially for $|t| \rightarrow \infty$. For $t \rightarrow 0$ it either remains finite or diverges logarithmically.

Proof. The Assumptions 3.7 on the spectral density $J$ translate into analyticity and other properties of $\hat{\gamma}$. We summarize them in an

Assertion. There are two constants $0<\psi<\pi / 2$ and $0<\eta<\delta$ such that $\hat{\gamma}$ can be continued analytically from the upper complex half-plane to the sector $\mathcal{H}_{\psi, \eta}:=\mathcal{H}_{\psi, \eta}^{-} \cup\{z \in \mathbb{C}: \operatorname{Im} z \geq 0\}$. Moreover,

$\sup _{z \in \mathcal{H}_{\psi, \eta}}|z \hat{\gamma}(z)|<\infty$

and the complex function

$z \mapsto \omega^{2}-z^{2}-\mathrm{i} z \hat{\gamma}(z) / m$

has no zeros in $\mathcal{H}_{\psi, \eta}$-except for the case $\omega=0$ in which $z=0$ is a simple zero.

Before we show the validity of the Assertion, we demonstrate how it can be exploited to prove the Statement. Consider the inverse Laplace transformation of the functions $\hat{\dot{G}}(z)=-\mathrm{i} z \hat{G}(z), \hat{\ddot{G}}(z)=-z^{2} \hat{G}(z)-1$ and $\hat{G}(z)$ (in the case $\omega>0$ ), respectively $\hat{G}(z)+(\mathrm{i} z \hat{\gamma}(0) / m)^{-1}$ (in the case $\left.\omega=0\right)$. For the last function to make sense, observe that $\hat{\gamma}(0)=\pi J(0)>0$ due to Assumption 3.7.i). According to the Assertion each of these functions is analytic in $\mathcal{H}_{\psi, \eta}$, remains bounded for $z \rightarrow-\mathrm{i} \eta$ and decays like $\mathcal{O}\left(\left|z^{-1}\right|\right)$ for $|z| \rightarrow \infty$, $z \in \mathcal{H}_{\psi, \eta}$. Therefore Cauchy's theorem allows to deform the integration 
contour in the inverse Laplace transformation from the upper complex halfplane to the boundary $\partial \mathcal{H}_{\psi, \eta}$. But now we are in the situation of the Abelian theorem in Doetsch (1950, Chap. 15, §4, Thm. 2), and (3.40)-(3.42) follow.

To prove the above assertion observe that

$\hat{\gamma}(z):=\mathrm{i} \int_{\partial \mathcal{H}_{\psi, \delta}} \mathrm{d} z^{\prime} \frac{J\left(z^{\prime}\right)}{z-z^{\prime}}, \quad z \in \mathcal{H}_{\psi, \eta}, 0<\eta<\delta$,

coincides in the upper half-plane $\operatorname{Im} z>0$ with the former definition (see (3.23) in the macroscopic limit)

$\hat{\gamma}(z)=\int_{0}^{\infty} \mathrm{d} t \mathrm{e}^{\mathrm{i} z t} \gamma(t)=\mathrm{i} \int_{\mathbb{R}} \mathrm{d} \nu \frac{J(\nu)}{z-\nu}$.

This is due to Assumptions 3.7.ii) and iii) and Cauchy's theorem. Moreover, for $z_{0} \in \mathcal{H}_{\psi, \eta}$ the difference quotient $\left(\hat{\gamma}(z)-\hat{\gamma}\left(z_{0}\right)\right) /\left(z-z_{0}\right)$ exists in the limit $z \rightarrow z_{0}$ by Assumption 3.7.iii) and dominated convergence. Hence, (3.45) provides the desired analytic continuation of $\hat{\gamma}$. The decay property (3.43) is evident from (3.45) and Assumption 3.7.iii). A necessary condition for a zero of (3.44) - except for the zero $z=0$ in the case $\omega=0$-is $\operatorname{Im} z<0$ as follows from Remark 3.5.i), $\operatorname{Re} \hat{\gamma}(\nu)=\pi J(\nu)>0$ for $\nu \in \mathbb{R}$ and Assumption 3.7.i). Furthermore, (3.43) implies that all zeros lie in a bounded region of $\mathcal{H}_{\psi, \eta}^{-}$. Hence, there exist only finitely many of them, because otherwise they would have a limit point, and by the identity theorem for analytic functions the function (3.44) would have to vanish for all $z \in \mathcal{H}_{\psi, \eta}$. One can therefore choose $\psi$ and $\eta$ sufficiently small such that there are no zeros at all in $\mathcal{H}_{\psi, \eta}$. This completes the proof of the Assertion.

It remains to prove the properties of the covariance function $\Phi_{\beta}$. Because of

$\Phi_{\beta}(-t)=\Phi_{\beta}(t)=\hbar \operatorname{Re}\left\{\int_{0}^{\infty} \mathrm{d} \nu J(\nu) \nu \operatorname{coth}(\beta \hbar \nu / 2) \mathrm{e}^{-\mathrm{i} \nu t}\right\}$

we assume $t \geq 0$ from now on. As a consequence of (3.33), $J(\nu)$ either decays like $\nu^{-2}$ for $\nu \rightarrow \infty$ or it is of the order $\mathcal{O}\left(\nu^{-2-r}\right)$ for some $r>0$. In the latter case the integral (3.47) exists as a Lebesgue integral for all $t \geq 0$ rendering $\Phi_{\beta}$ a bounded function. In the former case the integral (3.47) exists as an improper Riemann integral for $t>0$, and $\Phi_{\beta}$ has a singularity at $t=0$. In order to characterize this singularity, fix some $s>0$, set $L:=\lim _{\nu \rightarrow \infty}\left(\nu^{2} J(\nu)\right)$ and make the decomposition

$J(\nu) \nu \operatorname{coth}(\beta \hbar \nu / 2)=: \frac{L}{\nu}-\frac{L}{\nu} \mathrm{e}^{-s \nu}+f(\nu)$

which defines the Lebesgue integrable function $f$ over $[0, \infty[$. Hence, $f$ does not contribute to the singularity of $\Phi_{\beta}$ at $t=0$. The singularity of $\Phi_{\beta}$ comes solely from

$\int_{0}^{\infty} \mathrm{d} \nu\left(\frac{L}{\nu}-\frac{L}{\nu} \mathrm{e}^{-s \nu}\right) \mathrm{e}^{-\mathrm{i} \nu t}$ 
and the Abelian theorem in Doetsch (1950, Chap. 13, §3, Thm. 1) reveals that it is logarithmic.

In order to derive the exponential decay of $\Phi_{\beta}$ for $t \rightarrow \infty$ we proceed as in the case of the Green function. Analyticity and decay of $J$ in $\mathcal{H}_{\psi, \eta}^{-}$imply together with Cauchy's theorem the representation

$$
\begin{aligned}
\Phi_{\beta}(t) & =(\hbar / 2) \int_{\mathbb{R}} \mathrm{d} \nu J(\nu) \nu \operatorname{coth}(\beta \hbar \nu / 2) \mathrm{e}^{-\mathrm{i} \nu t} \\
& =(\hbar / 2) \int_{\partial \mathcal{H}_{\psi, \eta}} \mathrm{d} z J(z) z \operatorname{coth}(\beta \hbar z / 2) \mathrm{e}^{-\mathrm{i} z t},
\end{aligned}
$$

which is valid for $t>0$ and $0<\eta<\min \{\delta, 2 \pi /(\beta \hbar)\}$. The Abelian theorem (Doetsch, 1950, Chap. 15, §4, Thm. 2) then yields the exponential decay of $\Phi_{\beta}$ for $t \rightarrow \infty$. Thus $\Phi_{\beta}$ is also seen to be Lebesgue integrable over $\mathbb{R}$.

To formulate the main result of this section, it is illuminating to study first the expectation value

$$
\left\langle\langle a\rangle_{\beta, J}:=\lim _{n \rightarrow \infty}\left\langle w^{(n)}, a\right\rangle\right.
$$

of a system observable $a$ with respect to the thermal equilibrium state of the deterministic part $H^{(n)}$ of the total Hamiltonian in the macroscopic limit of the heat bath. The scalar product in (3.51) was defined in (3.13). Recalling the effective temperatures (3.16), the Wigner density of the corresponding pre-limit state may be written as

$$
\begin{aligned}
w^{(n)}(\mathbf{p}, \mathbf{q}):=\frac{\operatorname{det}\left(\Omega \beta_{\mathrm{eff}}(\hbar \Omega)\right)}{(2 \pi)^{n+1}} \exp \left\{-\frac{1}{2} \mathbf{p} \cdot \mathrm{M}^{-1 / 2} \beta_{\mathrm{eff}}(\hbar \Omega) \mathrm{M}^{-1 / 2} \mathbf{p}\right. \\
\left.-\frac{1}{2} \mathbf{q} \cdot \mathrm{M}^{1 / 2} \Omega^{2} \beta_{\mathrm{eff}}(\hbar \Omega) \mathrm{M}^{1 / 2} \mathbf{q}\right\} .
\end{aligned}
$$

Statement 3.10 below compiles three equivalent expressions for the thermal expectation values $\left\langle\left\langle p^{2}\right\rangle_{\beta, J}\right.$ and $\left\langle q^{2}\right\rangle_{\beta, J}$, which are available in the literature, see for example Haake and Reibold (1985), Ford et al. (1988b), Grabert et al. (1988, Table 2) and Weiss (1993, Chap. 6). Nevertheless we will prove them, since Statement 3.6 provides a quick way to do so.

Statement 3.10. The following identities hold under the Assumptions 3.7 on the spectral density:

$$
\begin{aligned}
\left\langle p^{2}\right\rangle_{\beta, J} & =\frac{\hbar}{2} \int_{\mathbb{R}} \mathrm{d} \nu J(\nu) \nu^{3}|\hat{G}(\nu)|^{2} \operatorname{coth}(\beta \hbar \nu / 2) \\
& =\frac{m \hbar}{2 \pi \mathrm{i}} \operatorname{PV} \int_{\mathbb{R}} \mathrm{d} \nu\left(\nu^{2} \hat{G}(\nu)+1\right) \operatorname{coth}(\beta \hbar \nu / 2) \\
& =\frac{m}{\beta} \sum_{l=-\infty}^{+\infty}\left(1-\nu_{l}^{2} \hat{G}\left(\mathrm{i}\left|\nu_{l}\right|\right)\right)
\end{aligned}
$$


and, if $\omega>0$,

$$
\begin{aligned}
\left\langle q^{2}\right\rangle_{\beta, J} & =\frac{\hbar}{2 m^{2}} \int_{\mathbb{R}} \mathrm{d} \nu J(\nu) \nu|\hat{G}(\nu)|^{2} \operatorname{coth}(\beta \hbar \nu / 2) \\
& =\frac{\hbar}{2 \pi \mathrm{i} m} \operatorname{PV} \int_{\mathbb{R}} \mathrm{d} \nu \hat{G}(\nu) \operatorname{coth}(\beta \hbar \nu / 2) \\
& =\frac{1}{m \beta} \sum_{l=-\infty}^{+\infty} \hat{G}\left(\mathrm{i}\left|\nu_{l}\right|\right) .
\end{aligned}
$$

Here, $\nu_{l}:=2 \pi l /(\beta \hbar)$ are the even Matsubara frequencies, $\hat{G}(\nu)$ denotes the analytic continuation of $\hat{G}(z)$ to the real axis, and the principal-value prescription in the middle lines of (3.53a) and (3.53b) has to be applied at the origin.

Remark 3.11. As is indicated by the notation, the expectation values $\left\langle\left\langle p^{2}\right\rangle_{\beta, J}\right.$ and $\left\langle\left\langle q^{2}\right\rangle_{\beta, J}\right.$ depend on the inverse temperature $\beta$ and on the spectral density $J$ of the heat bath. Moreover, they do also depend on Planck's constant $\hbar$. From the series representations in (3.53) one can derive the following chain of inequalities

$$
\begin{aligned}
\max \left\{\frac{1}{2 \beta}, \frac{\left(\omega / \omega_{J}\right)^{2}}{2 \beta_{\mathrm{eff}}\left(\hbar \omega_{J}\right)}\right\} & \leq \frac{m \omega^{2}}{2}\left\langle\left\langle q^{2}\right\rangle_{\beta, J} \leq \frac{1}{2 \beta_{\mathrm{eff}}(\hbar \omega)}\right. \\
& \leq \frac{1}{2 m}\left\langle\left\langle p^{2}\right\rangle_{\beta, J} \leq \frac{1}{2 \beta_{\mathrm{eff}}\left(\hbar \omega_{J}\right)}\right.
\end{aligned}
$$

where $\omega_{J}:=\sqrt{\omega^{2}+\gamma(0) / m}$ and $\gamma(0)=\int_{\mathbb{R}} \mathrm{d} \nu J(\nu)$. As a consequence, one can easily deduce the values of $\left\langle\left\langle p^{2}\right\rangle_{\beta, J} / 2 m\right.$ and $m \omega^{2}\left\langle\left\langle q^{2}\right\rangle_{\beta, J} / 2\right.$ in the lowfriction limit $J \searrow 0$ or the classical limit $\hbar \searrow 0$. For $J \searrow 0$ both expectation values tend to the quantum-mechanical canonical equilibrium value $1 / 2 \beta_{\text {eff }}(\hbar \omega)$ with respect to the harmonic-oscillator Hamiltonian $p^{2} /(2 m)+\left(m \omega^{2} / 2\right) q^{2}$. For $\hbar \searrow 0$ they tend to $1 / 2 \beta$ for all admissible $J$, as dictated by the equipartition theorem. The latter expression emerges also in the quantum-mechanical case $\hbar \neq 0$ as an asymptotic value for high temperatures $\beta \searrow 0$.

Proof of Statement 3.10. In order to calculate $\left\langle\left\langle p^{2}\right\rangle_{\beta, J}\right.$ one has to consider the macroscopic limit of the expectation value

$$
\left\langle w^{(n)}, p^{2}\right\rangle=\frac{m \hbar}{2}(\Omega \operatorname{coth}(\beta \hbar \Omega / 2))_{00} .
$$

Before doing this we rewrite the right-hand side of (3.55) with the help of Statement 3.6. To this end, observe that the function

$$
z \mapsto f(z)=\frac{z}{1+\lambda^{-2} z^{2}} \operatorname{coth}(\beta \hbar z / 2)
$$


fulfills the assumptions of Statement 3.6 for all $0<\varepsilon<2 \pi /(\beta \hbar)<\lambda$. Hence,

$$
\begin{aligned}
\left\langle w^{(n)}, p^{2}\right\rangle= & \frac{m \hbar}{2 \pi \mathrm{i}} \lim _{\lambda \rightarrow \infty} \int_{\mathbb{R}+\mathrm{i} \varepsilon} \mathrm{d} z \frac{1}{1+\lambda^{-2} z^{2}} z^{2} \hat{G}_{n}(z) \operatorname{coth}(\beta \hbar z / 2) \\
= & \frac{m \hbar}{2 \pi \mathrm{i}} \int_{\mathbb{R}+\mathrm{i} \varepsilon} \mathrm{d} z\left(z^{2} \hat{G}_{n}(z)+1\right) \operatorname{coth}(\beta \hbar z / 2) \\
& -\frac{m \hbar}{2 \pi \mathrm{i}} \lim _{\lambda \rightarrow \infty} \int_{\mathbb{R}+\mathrm{i} \varepsilon} \mathrm{d} z \frac{\operatorname{coth}(\beta \hbar z / 2)}{1+\lambda^{-2} z^{2}}
\end{aligned}
$$

where we have taken advantage of the fact that $z^{2} \hat{G}_{n}(z)+1$ falls of like $|z|^{-2}$ for $|z| \rightarrow \infty$. Thanks to

$$
\begin{aligned}
\int_{\mathbb{R}+\mathrm{i} \varepsilon} \mathrm{d} z \frac{\operatorname{coth}(\beta \hbar z / 2)}{1+\lambda^{-2} z^{2}} & =\mathrm{PV} \int_{\mathbb{R}} \mathrm{d} \nu \frac{\operatorname{coth}(\beta \hbar \nu / 2)}{1+\lambda^{-2} \nu^{2}}-\pi \mathrm{i} \operatorname{Res}_{z=0}\{\operatorname{coth}(\beta \hbar z / 2)\} \\
& =-2 \pi \mathrm{i} /(\beta \hbar)
\end{aligned}
$$

we conclude

$$
\begin{aligned}
\left\langle p^{2}\right\rangle_{\beta, J} & =\frac{m \hbar}{2 \pi \mathrm{i}}\left\{\int_{\mathbb{R}+\mathrm{i} \varepsilon} \mathrm{d} z\left(z^{2} \hat{G}(z)+1\right) \operatorname{coth}(\beta \hbar z / 2)+2 \pi \mathrm{i} /(\beta \hbar)\right\} \\
& =\frac{m \hbar}{2 \pi \mathrm{i}} \mathrm{PV} \int_{\mathbb{R}} \mathrm{d} \nu\left(\nu^{2} \hat{G}(\nu)+1\right) \operatorname{coth}(\beta \hbar \nu / 2) .
\end{aligned}
$$

The principal-value prescription has to be taken at the origin, and $\hat{G}(\nu)$ denotes the analytic continuation of $\hat{G}(z)$ to the real axis. This continuation exists due to Assumptions 3.7. The integral representation in the top line of (3.53a) follows from (3.59) by using the antisymmetry of the hyperbolic cotangent, the reality condition $\hat{G}(-\nu)=(\hat{G}(\nu))^{*}$ and $\operatorname{Im} \hat{G}(\nu)=$ $\pi m^{-1} J(\nu) \nu|\hat{G}(\nu)|^{2}$. The series representation in the bottom line of (3.53a) follows from evaluating (3.59) with the help of the residue theorem and the Mittag-Leffler expansion of the hyperbolic cotangent

$\operatorname{coth}(\beta \hbar \nu / 2)=\frac{2 \nu}{\beta \hbar} \sum_{l=-\infty}^{+\infty} \frac{1}{\nu^{2}+\nu_{l}^{2}}$.

Here, $\nu_{l}:=2 \pi l /(\beta \hbar)$ are the even Matsubara frequencies. Note that the pole for $l=0$, which lies on the integration contour, counts only half due to the principal-value prescription.

In order for $\left\langle\left\langle q^{2}\right\rangle_{\beta, J}\right.$ to exist we assume $\omega>0$ and start out with

$\left\langle w^{(n)}, q^{2}\right\rangle=\frac{\hbar}{2 m}\left(\frac{\operatorname{coth}(\beta \hbar \Omega / 2)}{\Omega}\right)_{00}$.

We pick three constants $0<\varepsilon<\lambda<\tilde{\varepsilon}<2 \pi /(\beta \hbar)$ and apply Statement 3.6 to the function

$z \mapsto f(z)=\frac{z}{\lambda^{2}+z^{2}} \operatorname{coth}(\beta \hbar z / 2)$ 
which gives

$$
\begin{aligned}
&\left\langle w^{(n)}, q^{2}\right\rangle= \frac{\hbar}{2 \pi \mathrm{i} m} \lim _{\lambda \searrow 0} \int_{\mathbb{R}+\mathrm{i} \varepsilon} \mathrm{d} z \frac{z^{2}}{\lambda^{2}+z^{2}} \hat{G}_{n}(z) \operatorname{coth}(\beta \hbar z / 2) \\
&=\frac{\hbar}{2 \pi \mathrm{i} m} \lim _{\lambda \searrow 0}\left\{\int_{\mathbb{R}+\mathrm{i} \tilde{\varepsilon}} \mathrm{d} z \frac{z^{2}}{\lambda^{2}+z^{2}} \hat{G}_{n}(z) \operatorname{coth}(\beta \hbar z / 2)\right. \\
&\left.\quad+\pi \mathrm{i} \lambda \hat{G}_{n}(\mathrm{i} \lambda) \cot (\beta \hbar \lambda / 2)\right\} \\
&=\frac{\hbar}{2 \pi \mathrm{i} m}\left\{\int_{\mathbb{R}+\mathrm{i} \tilde{\varepsilon}} \mathrm{d} z \hat{G}_{n}(z) \operatorname{coth}(\beta \hbar z / 2)+\frac{2 \pi \mathrm{i}}{\beta \hbar} \hat{G}_{n}(0)\right\} .
\end{aligned}
$$

The second equality in (3.63) follows from deforming the integration contour across the pole at $z=\mathrm{i} \lambda$. Performing the macroscopic limit $n \rightarrow \infty$ and subsequently the limit $\tilde{\varepsilon} \searrow 0$, one arrives at

$$
\left\langle\left\langle q^{2}\right\rangle_{\beta, J}=\frac{\hbar}{2 \pi \mathrm{i} m} \mathrm{PV} \int_{\mathbb{R}} \mathrm{d} \nu \hat{G}(\nu) \operatorname{coth}(\beta \hbar \nu / 2) .\right.
$$

The integral representation in the top line of $(3.53 \mathrm{~b})$ and the series representation in the bottom line of (3.53b) are derived from (3.64) in the same way as was demonstrated for $\left\langle\left\langle p^{2}\right\rangle_{\beta, J}\right.$.

As the main result of this section, we describe the spreading of states under the reduced averaged dynamics of Model 3.1 in the long-time limit.

Statement 3.12. If the spectral density $J$ of the macroscopic heat bath satisfies Assumptions 3.7, then the second moment of the particle momentum approaches a finite value under the reduced averaged dynamics (3.36) of Model 3.1

$\lim _{t \rightarrow \infty}\left(\mathcal{R}_{t}^{(\beta, J)}\right)^{\dagger} p^{2}=\left\langle\left\langle p^{2}\right\rangle\right\rangle_{\beta, J}+\left(-\partial_{q}^{2} C\right)(0) \int_{\mathbb{R}} \frac{\mathrm{d} \nu}{2 \pi} \nu^{2}|\hat{G}(\nu)|^{2}$.

In the harmonically bound case $\omega>0$, the same is true for the second moment of position

$\lim _{t \rightarrow \infty}\left(\mathcal{R}_{t}^{(\beta, J)}\right)^{\dagger} q^{2}=\left\langle\left\langle q^{2}\right\rangle_{\beta, J}+\frac{\left(-\partial_{q}^{2} C\right)(0)}{m^{2}} \int_{\mathbb{R}} \frac{\mathrm{d} \nu}{2 \pi}|\hat{G}(\nu)|^{2}\right.$.

In the unbound case $\omega=0$ the limit (3.66) is to be replaced by the diffusive behaviour

$\lim _{t \rightarrow \infty} \frac{\left(\mathcal{R}_{t}^{(\beta, J)}\right)^{\dagger} q^{2}}{t}=\frac{2}{\pi J(0)}\left(\beta^{-1}+\frac{\left(-\partial_{q}^{2} C\right)(0)}{2 \pi J(0)}\right)$.

Above we have used the thermal expectation values (3.53). The results imply in particular that the mean energy $\left(\mathcal{R}_{t}^{(\beta, J)}\right)^{\dagger}\left(p^{2} /(2 m)+\left(m \omega^{2} / 2\right) q^{2}\right)$, respectively $\left(\mathcal{R}_{t}^{(\beta, J)}\right)^{\dagger} p^{2} /(2 m)$ in the unbound case $\omega=0$, remains finite in the long-time limit. 
Remarks 3.13. i) The right-hand sides of (3.65) - (3.67) are constant functions on phase space $\mathbb{R} \times \mathbb{R}$. Consequently, the asymptotic spreading of states is universal in the sense that it does not depend on the initial state.

ii) The terms which are due to the white noise in (3.65) - (3.67) depend only on the curvature of its covariance function at the origin and, via the Green function $G$, on the spectral density $J$ of the heat bath. On the other hand, the thermal equilibrium expectation values $\left\langle\left\langle p^{2}\right\rangle_{\beta, J}\right.$ and $\left\langle\left\langle q^{2}\right\rangle_{\beta, J}\right.$ inhibit also a dependence on Planck's constant. But it is only in the subsequent low-friction limit $J \searrow 0$ that they reduce to the quantum-mechanical canonical equilibrium values with respect to the system Hamiltonian alone, see Remark 3.11 and confer also Ford et al. (1965), Davies (1973), Benguria and Kac (1981) and Maassen (1984).

iii) In Jayannavar and Kumar (1982) and Heinrichs (1983a) it has already been conjectured that the interplay of white noise and dissipation would lead to a diffusive spreading in position if $\omega=0$. Eq. (3.67) provides the corresponding quantitative statement. It is to be compared with the $t^{3}$-behaviour $(2.60 \mathrm{e})$ of $\mathcal{T}_{t}^{\dagger} q^{2}$ in the situation without dissipation. The $\hbar$-independence of the diffusion constant on the right-hand side of (3.67) reveals the classical character of this phenomenon.

$i v$ ) In the absence of white noise, $C(0)=0$, Eqs. (3.65) - (3.67) reduce to known results for the damped harmonic oscillator $(\omega>0)$ and the damped free particle $(\omega=0)$, respectively. In particluar, the diffusion constant on the right-hand side of (3.67) is then given by an Einstein type of relation (Sutherland, 1905; Einstein, 1905; Kubo et al., 1991). In the above statement we have given precise and explicit assumptions on the spectral density under which these results are valid. At zero temperature, $\beta=\infty$, or for a spectral density with $J(0)=0$ or $J(0)=$ $\infty$, which is excluded by our assumptions, non-exponential relaxation and anomalous diffusion is expected, see for example Haake and Reibold (1985), Grabert et al. (1988) and Weiss (1993, Chap. 6).

Proof of Statement 3.12. Statement 3.9 implies that the first term on the right-hand side of (3.37a) and (3.37b), respectively, does not contribute to the leading behaviour in the limit $t \rightarrow \infty$. For the case $\omega=0$ the leading behaviour of $(3.37 \mathrm{~b})$ is determined by the limit (3.40). The result (3.67) follows from it with the help of the relation

$\int_{\mathbb{R}} \mathrm{d} t \Phi_{\beta}(t)=2 \pi \beta^{-1} J(0)$.

The integral $\int_{0}^{t} \mathrm{~d} s(\dot{G}(s))^{2}$ in (3.37a) converges for $t \rightarrow \infty$. For $\omega>0$ the same is true for the integral $\int_{0}^{t} \mathrm{~d} s(G(s))^{2}$ in (3.37b). Using Parseval's 
formula we thus conclude

$\int_{0}^{\infty} \mathrm{d} s(\dot{G}(s))^{2}=\int_{\mathbb{R}} \frac{\mathrm{d} \nu}{2 \pi} \nu^{2}|\hat{G}(\nu)|^{2}$

and, if $\omega>0$,

$\int_{0}^{\infty} \mathrm{d} s(G(s))^{2}=\int_{\mathbb{R}} \frac{\mathrm{d} \nu}{2 \pi}|\hat{G}(\nu)|^{2}$.

This explains the last term in (3.65) and (3.66), respectively. Finally, the convolution theorem, Definition (3.34) and Statement 3.10 imply

$$
\begin{aligned}
\int_{0}^{\infty} \mathrm{d} s \int_{0}^{\infty} \mathrm{d} s^{\prime} & \dot{G}(s) \Phi_{\beta}\left(s-s^{\prime}\right) \dot{G}\left(s^{\prime}\right) \\
& =\frac{\hbar}{2} \int_{\mathbb{R}} \mathrm{d} \nu J(\nu) \nu^{3}|\hat{G}(\nu)|^{2} \operatorname{coth}(\beta \hbar \nu / 2) \\
& =\left\langle\left\langle p^{2}\right\rangle_{\beta, J}\right.
\end{aligned}
$$

and, if $\omega>0$,

$$
\begin{aligned}
m^{-2} \int_{0}^{\infty} \mathrm{d} s \int_{0}^{\infty} \mathrm{d} s^{\prime} G(s) \Phi_{\beta}\left(s-s^{\prime}\right) G\left(s^{\prime}\right) \\
=\frac{\hbar}{2 m^{2}} \int_{\mathbb{R}} \mathrm{d} \nu J(\nu) \nu|\hat{G}(\nu)|^{2} \operatorname{coth}(\beta \hbar \nu / 2) \\
=\left\langle\left\langle q^{2}\right\rangle_{\beta, J} .\right.
\end{aligned}
$$

\section{Outlook}

The present paper is devoted to the averaged dynamics of white-noise systems. Natural extensions of this work concern the fluctuations around the averaged dynamics and the effects of a non-vanishing correlation time, that is, coloured-noise perturbations. Both problems have not been treated satisfactorily so far. Diverse attempts, both analytical (but based on uncontrolled approximations) and numerical, can be found in the literature. To our knowledge, all of them treat only the case without dissipation. Unfortunately, their results are not free from contradictions. It may therefore be of help to compile some of these references and to relate their findings.

In the preceding sections we analyzed the averaged dynamics $w(p, q) \mapsto$ $\overline{w_{t}(p, q)}$ in order to study the average $\overline{\left\langle w_{t}, a\right\rangle}$ of the quantum-mechanical expectation value $\left\langle w_{t}, a\right\rangle$ of an observable $a$ at time $t$. Fluctuations around

this average are described by the higher moments $\overline{\left\langle w_{t}, a\right\rangle^{N}}, N \geq 2$, for which Jensen's inequality

$$
\overline{\left\langle w_{t}, a\right\rangle^{N}} \geq\left(\overline{\left\langle w_{t}, a\right\rangle}\right)^{N}
$$


provides a (generally rather poor) lower bound. The appropriate tool for studying the $N$-th moment $\overline{\left\langle w_{t}, a\right\rangle^{N}}$ is the averaged dynamics of a noninteracting $N$-particle white-noise system. In particular, it provides the relevant information about the mapping $w\left(p_{1}, q_{1}\right) \cdot \ldots \cdot w\left(p_{N}, q_{N}\right) \mapsto$ $\overline{w_{t}\left(p_{1}, q_{1}\right) \cdot \ldots \cdot w_{t}\left(p_{N}, q_{N}\right)}$. This dynamics is again given by a semigroup, whose generator can be constructed explicitly. However, since one has not succeeded in representing the semigroup as in Statement 2.5, which was the starting point for all the exact calculations, one had to resort to approximations. Stintzing (1992) has carried out an analysis up to second-order perturbation theory for $N=2$ particles on a lattice with nearest-neighbour hopping and a site-diagonal white-noise perturbation. Taking the lattice position $\vec{q}=\left(q_{1}, \ldots, q_{d}\right)$ as the observable, he finds an asymptotic result which, in our notation, would read

$\overline{\left\langle w_{t}, \vec{q}\right\rangle^{2}} \sim t^{0} \quad$ for $\quad t \rightarrow \infty$.

This result is shown to be independent of the number $d$ of space dimensions of the lattice. By appealing to an analogy to a classical master equation, he even claims that (4.2) represents the exact asymptotic behaviour. However, (4.2) should be contrasted with the numerical calculations of Bouchaud et al. (1992) and Saul et al. (1992) which suggest a result different from (4.2)

$\overline{\left\langle w_{t}, \vec{q}\right\rangle^{2}} \sim\left\{\begin{array}{ll}t^{1 / 2}, & d=1,2 \\ \ln t, & d=3\end{array} \quad\right.$ for $\quad t \rightarrow \infty$.

Other aspects of the averaged dynamics of many-particle white-noise systems can be found in Girvin and Mahan (1979) and Jauho (1987).

Coloured-noise perturbations have attracted much attention in the last decade. On the one hand they give rise to less idealized models, on the other hand they provide a variety of interesting features due to the existence of an additional time scale, the correlation time $\tau$ of the noise. Because of this complexity, there are only few exact analytical results available. Moreover, these are limited to models in which the noise has some additional nice properties. We only mention a Markovian noise in time (Cheremshantsev, 1982; Pillet, 1985, 1986; Tcheremchantsev, 1997), rank-one perturbations with coloured-noise coupling coefficients (Chvosta, 1991; Neu and Speicher, 1994) and coloured-noise couplings to some deterministic external force (Kitahara and Haus, 1979; Inaba, 1981). For classical systems, however, there exists a more detailed picture, see for example the review by Hänggi and Jung (1995). Quantum-mechanical models similar to ours with a Gaussian coloured-noise have been studied by Golubović et al. (1991), Rosenbluth (1992), Heinrichs (1992), Lebedev et al. (1995) and Heinrichs (1996). The starting point for the calculations in Golubović et al. (1991) is the corresponding classical model which is analyzed within the Martin-Siggia-Rose formalism. After performing a short-time expansion they derive the asymp- 
totic long-time behaviour

$\overline{\left\langle w_{t}, \vec{p}^{2}\right\rangle} \sim \begin{cases}t^{2 / 5}, & d=1 \\ t^{1 / 2}, & d \geq 2\end{cases}$

$\overline{\left\langle w_{t}, \vec{q}^{2}\right\rangle} \sim \begin{cases}t^{12 / 5}, & d=1 \\ t^{9 / 4}, & d \geq 2\end{cases}$

for $t \rightarrow \infty$,

which, according to a quasi-classical argument, should not be modified by quantum fluctuations. Golubović et al. (1991) also perform a numerical simulation in order to support (4.4). In a Comment, Rosenbluth (1992) criticizes the derivation of (4.4), as it does not guarantee the conservation of probability. His alternative derivation, which is not presented in detail, leads to a modification of (4.4) in more than one space dimension

$\overline{\left\langle w_{t}, \vec{p}^{2}\right\rangle} \sim \quad t^{2 / 5}$

$\overline{\left\langle w_{t}, \vec{q}^{2}\right\rangle} \sim \begin{cases}t^{12 / 5}, & d=1 \\ t^{2}, & d \geq 2\end{cases}$

A genuine quantum-mechanical treatment of the model is given by Heinrichs (1992). He succeeds in averaging the von Neumann equation up to first order in $\tau / t$ yielding

$\overline{\left\langle w_{t}, \vec{p}^{2}\right\rangle} \sim t\left\{1+\mathcal{O}\left((\tau / t)^{2}\right)\right\}$

$\overline{\left\langle w_{t}, \vec{q}^{2}\right\rangle} \sim t^{3}\left\{1+\frac{3}{2}(\tau / t)+\mathcal{O}\left((\tau / t)^{2}\right)\right\}$

for $t \rightarrow \infty$.

Using a similar method, Heinrichs (1996) also investigates the corresponding behaviour at intermediate times. Lebedev et al. (1995) argue on rather speculative grounds that (4.4) should be correct up to very long times, but in the limit $t \rightarrow \infty$ the exponents should be replaced by smaller ones.

We conclude that the effects of a non-vanishing correlation time are controversially discussed at present. In order to arrive at a unifying picture, it seems that suitable bounds and other controlled approximations to the averaged dynamics are needed.

\section{Appendix A The Weyl-Wigner-Moyal representation}

In this Appendix we intend to give a brief overview of the linear phase-space representation of quantum mechanics which dates back to Weyl (1928), Wigner (1932) and Moyal (1949). More detailed information and proofs of the results mentioned here can be found in the review articles Tatarskiı (1983), Balasz and Jennings (1984) and Hillary et al. (1984). Extensions of the formalism in order to describe many-particle systems are dealt with in Gadella 
and Nieto $(1993,1994)$, extensions to a non-Euclidean phase space in Bayen et al. (1978) and Kasperkovitz and Peev (1994). Some more mathematical issues are contained in Pool (1966), Daubechies (1980, 1983), Folland (1989), Lieb (1990) and Fedosov (1996). The articles Molzahn and Osborn (1994), Osborn and Molzahn (1995), Arai (1995) and Robinson (1993) are concerned with quasi-classical properties and the classical limit.

For simplicity we will restrict ourselves to describe a single spinless quantum particle which has the Euclidean line $\mathbb{R}$ as its configuration space. The generalization to several Cartesian degrees of freedom is merely a matter of notation. In the standard Hilbert-space formulation states and observables of the particle are represented by certain linear operators on $L^{2}(\mathbb{R})$, the Hilbert space of Lebesgue square-integrable complex-valued functions on $\mathbb{R}$. The link to phase space is provided by

Definition A.1. Let $F$ be a linear operator on $\mathrm{L}^{2}(\mathbb{R})$. The mapping $\mathcal{W}$ : $F \mapsto \mathcal{W}(F):=f$,

$f: \mathbb{R} \times \mathbb{R} \rightarrow \mathbb{C}, \quad f(p, q):=\int_{\mathbb{R}} \mathrm{d} r \mathrm{e}^{\mathrm{i} p r / \hbar}\langle q-r / 2|F| q+r / 2\rangle$

is called Weyl-Wigner mapping. The phase-space function $f$ is called the Weyl-Wigner symbol of the operator F. In (A1) we have used the Dirac notation for the integral kernel of an operator in the position representation.

Pool (1966) shows that the Weyl-Wigner mapping induces an isometric isomorphism between the space of Hilbert-Schmidt operators on $L^{2}(\mathbb{R})$ and the Hilbert space $L^{2}(\mathbb{R} \times \mathbb{R})$ of square-integrable complex-valued functions on phase space. For an extension of (A1) to the Banach space of bounded operators on $L^{2}(\mathbb{R})$, see Daubechies (1980). For the purpose of the present paper we may safely ignore these more subtle issues, and we will use (A1) for all operators $F$ for which it makes sense, possibly in a distributional one.

Some important properties of the Weyl-Wigner mapping are listed without proof in

Statement A.2. i) Compatibility with the complex-linear structure:

$\mathcal{W}\left(\alpha F+\beta G^{\dagger}\right)=\alpha \mathcal{W}(F)+\beta \mathcal{W}(G)^{*}=\alpha f+\beta g^{*}$,

where $\alpha, \beta \in \mathbb{C}, G^{\dagger}$ is the Hilbert adjoint of $G$ on $\mathrm{L}^{2}(\mathbb{R})$ and $g^{*}$ is the complex conjugate of $g$.

ii) Operator products correspond to star products of symbols (the arrows indicate on which symbol a particular differentiation is meant to act):

$\mathcal{W}(F G)=f \exp \left\{-\frac{\mathrm{i} \hbar}{2}\left(\overleftarrow{\partial}_{p} \vec{\partial}_{q}-\overleftarrow{\partial}_{q} \vec{\partial}_{p}\right)\right\} g=: f \star g$ 
iii) Isometry:

$\operatorname{Trace}\left(F^{\dagger} G\right)=(2 \pi \hbar)^{-1} \int_{\mathbb{R} \times \mathbb{R}} \mathrm{d} p \mathrm{~d} q f^{*}(p, q) g(p, q)=:(2 \pi \hbar)^{-1}\langle f, g\rangle$.

iv) Weyl quantization as the inverse mapping:

$$
\begin{aligned}
F=\mathcal{W}^{-1}(f) & =\int_{\mathbb{R} \times \mathbb{R}} \frac{\mathrm{d} p \mathrm{~d} q}{2 \pi \hbar} \mathrm{e}^{\mathrm{i}(p-P) q / \hbar} f(p, Q+q / 2) \\
& =\left.f\left(-\mathrm{i} \hbar \partial_{q^{\prime}},-\mathrm{i} \hbar \partial_{p^{\prime}}\right) \mathrm{e}^{\mathrm{i}\left(q^{\prime} P+p^{\prime} Q\right) / \hbar}\right|_{p^{\prime}=0=q^{\prime}} .
\end{aligned}
$$

Here $P$ and $Q$ denote the momentum and position operator, respectively.

v) Diagonal matrix elements by integration:

$$
\int_{\mathbb{R}} \frac{\mathrm{d} p}{2 \pi \hbar} f(p, q)=\langle q|F| q\rangle, \quad \int_{\mathbb{R}} \frac{\mathrm{d} q}{2 \pi \hbar} f(p, q)=\langle p|F| p\rangle .
$$

Remark A.3. According to Statement A.2.ii) the symbol of the standardized commutator is given by

$$
\begin{aligned}
\mathcal{W}((\mathrm{i} / \hbar)(F G-G F)) & =(\mathrm{i} / \hbar)(f \star g-g \star f) \\
& =f \frac{2}{\hbar} \sin \left\{\frac{\hbar}{2}\left(\overleftarrow{\partial}_{p} \vec{\partial}_{q}-\overleftarrow{\partial}_{q} \vec{\partial}_{p}\right)\right\} g \\
& =:[f, g] .
\end{aligned}
$$

It is called Moyal bracket. If either $f$ or $g$ is a quadratic polynomial in $p$ and $q$, then the Moyal bracket reduces to the Poisson bracket

$$
[f, g]=\left(\partial_{p} f\right)\left(\partial_{q} g\right)-\left(\partial_{q} f\right)\left(\partial_{p} g\right) \text {. }
$$

Regardless of this assumption on $f$ and $g$, the equality (A8) is always true in the classical limit $\hbar \searrow 0$.

There are uncountably many ways of associating a phase-space function with a quantum-mechanical operator and vice versa (Cohen, 1966; Agarwal and Wolf, 1970; Fink and Leschke, 1993). The Weyl-Wigner mapping, respectively the Weyl quantization, provides the one that treats position and momentum in a totally symmetric way. This is illustrated by the following Examples A.4. i) $\mathcal{W}^{-1}(f(q))=f(Q), \quad \mathcal{W}^{-1}(f(p))=f(P)$,
ii) $\mathcal{W}^{-1}(p f(q))=\frac{1}{2}(\operatorname{Pf}(Q)+f(Q) P)$,
iii) $\mathcal{W}(P f(Q))=p f(q)-(\mathrm{i} \hbar / 2)\left(\partial_{q} f\right)(q)$,
iv) $\mathcal{W}(P f(Q) P)=p^{2} f(q)+\left(\hbar^{2} / 4\right)\left(\partial_{q}^{2} f\right)(q)$. 
Phase-space functions which represent quantum-mechanical states are called Wigner densities. By convention we say that a phase-space function $w$ is a Wigner density if it can be written as

$w=(2 \pi \hbar)^{-1} \mathcal{W}(W)$,

where $W$ is some state operator, that is, $W=W^{\dagger} \geq 0$ and Trace $W=1$.

Statement A.5. Wigner densities are bounded, $|w(p, q)| \leq(\pi \hbar)^{-1}$, normalized, $\langle w, 1\rangle=1$, and square-integrable, $\langle w, w\rangle \leq(2 \pi \hbar)^{-1}$. Moreover, one has $\langle w, w\rangle=(2 \pi \hbar)^{-1}$ if and only if $w$ represents a pure state.

In contrast to probability densities, however, Wigner densities may also take on negative values. This fact is a consequence of Statements A.2.i) and $v$ ), see e.g. Wigner (1971). A simple example is provided by the pure state $W=|\psi\rangle\langle\psi|$ whose position amplitude $\langle q \mid \psi\rangle$ is the positive square root of a uniform probability density over some interval. This example shows in addition that Wigner densities need not be Lebesgue integrable (Daubechies, 1983).

Below we give two intrinsic characterizations of Wigner densities. The first one relies on the factorization $W=\left(W^{1 / 2}\right)^{2}$ (Shirokov, 1979), the second one reflects the corresponding properties of state operators (Narcowich and O'Connell, 1986).

Statement A.6. A phase-space function $w$ is a Wigner density, if and only if there exists a real-valued phase-space function $u$ with $\langle u, u\rangle=1$ such that $w=u \star u$.

Statement A.7. A phase-space function $w$ is a Wigner density, if and only if it has the following three properties:

1. $w=w^{*}$,

2. $\langle w, 1\rangle=1$,

3. $\langle w, v\rangle \geq 0$ for all Wigner densities $v$.

Statement A.6 supports that Wigner densities converge to probability densities in the classical limit $\hbar \searrow 0$ (at least in some weak sense). From a rigorous mathematical point of view, however, such questions seem to be highly nontrivial. This is due to severe technical difficulties which arise in part from the missing Lebesgue integrability of Wigner densities. Related aspects of the classical limit of quantum-mechanical phase-space representations may be found in Robinson (1993), Arai (1995) and Werner (1995).

Acknowledgements. It is a pleasure for Hajo Leschke to thank Wolfgang Kundt for guiding his first steps in theoretical physics with so much enthusiasm. In particular, he explained to him the Weyl-Wigner-Moyal representation some thirty years ago (Kundt, 1967). This work was supported by the Deutsche Forschungsgemeinschaft under grant number Le 330/10-1. 


\section{References}

G.S. Agarwal, E. Wolf, Calculus for functions of noncommuting operators and general phase-space methods in quantum mechanics I-III, Phys. Rev. D 2 (1970) 2161-2186, 2187-2205, 2206-2225

R. Alicki, K. Lendi, Quantum-dynamical semigroups and applications, Springer, Berlin 1987

P.W. Anderson, Absence of diffusion in certain random lattices, Phys. Rev. 109 (1958) 1492-1505

J. Anglin, S. Habib, Classical dynamics for linear systems: The case of quantum Brownian motion, Mod. Phys. Lett. A 11 (1996) 2655-2662

A. Arai, On a model of a harmonic oscillator coupled to a quantized, massless, scalar quantum field. I, II, J. Math. Phys. 22 (1981) 2539-2548, 2549-2552

T. Arai, Some extensions of semiclassical limit $\hbar \rightarrow 0$ for Wigner functions on phase space, J. Math. Phys. 36 (1995) 622-630

N.L. Balasz, B.K. Jennings, Wigner's function and other distribution functions on Mock phase spaces, Phys. Rep. 104 (1984) 347-391

T. Banks, L. Susskind, M.E. Peskin, Difficulties for the evolution of pure states into mixed states, Nucl. Phys. B 244 (1984) 125-134

H. Bauer, Probability theory, Walter de Gruyter, Berlin 1996. German original: 4th ed., Walter de Gruyter, Berlin 1991

R. Bausch, Bewegungsgesetze nicht abgeschlossener Quantensysteme, Z. Phys. 193 (1966) 246-265 (in German)

F. Bayen, M. Flato, C. Fronsdal, A. Lichnerowicz, D. Sternheimer, Deformation theory and quantization. I, II, Ann. Phys. (N.Y.) 111 (1978) 61-110, 111-151

J.S. Bell, Speakable and unspeakable in quantum mechanics, Cambridge University Press, Cambridge 1987

R. Benguria, M. Kac, Quantum Langevin equation, Phys. Rev. Lett. 46 (1981) 1-4

V.L. Bonch-Bruevich, R. Enderlein, B. Esser, R. Keiper, A.G. Mironov, I.P. Zvyagin, Elektronentheorie ungeordneter Halbleiter, VEB Deutscher Verlag der Wissenschaften, Berlin 1984 (in German). Russian original: Nauka, Moscow 1981

J.P. Bouchaud, D. Touati, D. Sornette, Waves in a rapidly varying random potential: a numerical study, Phys. Rev. Lett. 68 (1992) 1787-1790

Z. Brzeźniak, F. Flandoli, Almost sure approximation of Wong-Zakai type for stochastic partial differential equations, Stochastic Proc. Appl. 55 (1995) 329-358

A.O. Caldeira, A.J. Leggett, Path integral approach to quantum Brownian motion, Physica A 121 (1983) 587-616. Erratum: ibid. 130 (1985) 374

R. Carmona, J. Lacroix, Spectral theory of random Schrödinger operators, Birkhäuser, Boston 1990

S.E. Cheremshantsev, Averaging of the solution of the Schrödinger equation with a randomly time-dependent potential, Sov. Phys. Dokl. 27 (1982) 718-720. Russian original: Dokl. Akad. Nauk SSSR 266 (1982) 597-601

P. Chvosta, Exact solution of a stochastic dimer problem with single-site energy modulation, Physica A 178 (1991) 168-194

L. Cohen, Generalized phase-space distributions, J. Math. Phys. 7 (1966) 781-786

I. Daubechies, On the distributions corresponding to bounded operators in the Weyl correspondence, Commun. Math. Phys. 75 (1980) 229-238

I. Daubechies, Continuity statements and counterintuitive examples in connection with Weyl quantization, J. Math. Phys. 24 (1983) 1453-1461

E.B. Davies, The harmonic oscillator in a heat bath, Commun. Math. Phys. 33 (1973) 171-186

G. Doetsch, Handbuch der Laplace-Transformation, vol. 1, Birkhäuser, Basel 1950 (in German)

M.D. Donsker, On function space integrals, in W.T. Martin, I. Segal (eds.), Analysis in function space, The M.I.T. Press, Cambridge (Mass.) 1964, pp. 17-30

J.L. Doob, Stochastic processes, Wiley, New York 1964

G.V. Efimov, W. von Waldenfels, On the instability of an oscillator in a field, Ann. Phys. (N.Y.) 233 (1994) 182-213

A. Einstein, Über die von der molekularkinetischen Theorie der Wärme geforderte Bewegung von in ruhenden Flüssigkeiten suspendierten Teilchen, Ann. Physik (4. Folge) 17 
(1905) 549-560 (in German)

B. Fedosov, Deformation quantization and index theory, Akademie Verlag, Berlin 1996

D.K. Ferry, H.L. Grubin, Modeling of quantum transport in semiconductor devices, in H. Ehrenreich, F. Spaepen (eds.), Solid State Physics, vol. 49, Academic, San Diego 1995, pp. 283-448

R.P. Feynman, F.L. Vernon, Jr., The theory of a general quantum system interacting with a linear dissipative system, Ann. Phys. (N.Y.) 24 (1963) 118-173

H. Fink, H. Leschke, Phase-space- and other representations of quantum mechanics: a unifying approach, in H.D. Doebner, W. Scherer, F. Schroeck, Jr. (eds.), Classical and quantum systems - foundations and symmetries, World Scientific, Singapore 1993, pp. 416-419

W. Fischer, H. Leschke, P. Müller, Dynamics by white-noise Hamiltonians, Phys. Rev. Lett. 73 (1994) 1578-1581

G.B. Folland, Harmonic analysis in phase space, Princeton University Press, Princeton 1989

G.W. Ford, M. Kac, P. Mazur, Statistical mechanics of assemblies of coupled oscillators, J. Math. Phys. 6 (1965) 504-515

G.W. Ford, M. Kac, On the quantum Langevin equation, J. Stat. Phys. 46 (1987) 803-810

G.W. Ford, J.T. Lewis, R.F. O'Connell, Quantum Langevin equation, Phys. Rev. A 37 (1988a) 4419-4428

G.W. Ford, J.T. Lewis, R.F. O'Connell, Independent oscillator model of a heat bath: exact diagonalization of the Hamiltonian, J. Stat. Phys. 53 (1988b) 439-455

G.W. Ford, R.F. O'Connell, Derivative of the hyperbolic cotangent, Nature (London) $\mathbf{3 8 0}$ (1996) 113-114

K. Furutsu, On the statistical theory of electromagnetic waves in a fluctuating medium (1), J. Res. Nat. Bur. Stand. 67D (1963) 303-323

M. Gadella, L.M. Nieto, Fermion systems and the Moyal representation of quantum mechanics, J. Phys. A 26 (1993) 6043-6053

M. Gadella, L.M. Nieto, On the Moyal representation of quantum identical particles, Fortschr. Phys. 42 (1994) 261-279

C.W. Gardiner, Quantum noise, Springer, Berlin 1991

G.C. Ghirardi, A. Rimini, Old and new ideas in the theory of quantum measurement, in A.I. Miller (ed.), Sixty-two years of uncertainty, Plenum, New York 1990, pp. 167-191

G.C. Ghirardi, A. Rimini, T. Weber, Unified dynamics for microscopic and macroscopic systems, Phys. Rev. D 34 (1986) 470-491

G.C. Ghirardi, A. Rimini, T. Weber, Disentanglement of quantum wave functions: answer to "Comment on 'Unified dynamics for microscopic and macroscopic systems" ", Phys. Rev. D 36 (1987) 3287-3289

S.M. Girvin, G.D. Mahan, Exact results for the quantum diffusion of many particles in a stochastic medium, Phys. Rev. B 20 (1979) 4896-4901

J. Glimm, A. Jaffe, Quantum physics: a functional integral point of view, 2nd ed., Springer, New York 1987

L. Golubović, S. Feng, F.-A. Zeng, Classical and quantum superdiffusion in a timedependent random potential, Phys. Rev. Lett. 67 (1991) 2115-2118

V. Gorini, A. Kossakowski, E.C.G. Sudarshan, Completely positive dynamical semigroups of $N$-level systems, J. Math. Phys. 17 (1976) 821-825

H. Grabert, P. Schramm, G.-L. Ingold, Quantum Brownian motion: the functional integral approach, Phys. Rep. 168 (1988) 115-207

F. Haake, R. Reibold, Strong damping and low-temperature anomalies for the harmonic oscillator, Phys. Rev. A 32 (1985) 2462-2475

S. Habib, Quantum diffusion, preprint hep-th/9410181, 1994

P. Hänggi, P. Jung, Colored noise in dynamical systems, Adv. Chem. Phys. 89 (1995) 239-326

H. Haken, P. Reineker, The coupled coherent and incoherent motion of excitons and its influence on the line shape of optical absorption, Z. Phys. 249 (1972) 253-268

H. Haug, A.-P. Jauho, Quantum kinetics in transport and optics of semiconductors, Springer, Berlin 1996

S.W. Hawking, Breakdown of predictability in gravitational collapse, Phys. Rev. D 14 (1976) 2460-2473

S.W. Hawking, The unpredictability of quantum gravity, Commun. Math. Phys. 87 (1982) $395-414$ 
S.W. Hawking, Non-trivial topologies in quantum gravity, Nucl. Phys. B 244 (1984) 135146

J. Heinrichs, Quantum treatment of Brownian motion and influence of dissipation on diffusion in dynamically disordered systems, Z. Phys. B 50 (1983a) 269-273

J. Heinrichs, Continuum tight binding models and quantum transport in the presence of dynamical disorder, Z. Phys. B 53 (1983b) 175-181

J. Heinrichs, Diffusion and superdiffusion of a quantum particle in time-dependent random potentials, Z. Phys. B 89 (1992) 115-121. Erratum: ibid. 100 (1996) 327-328

J. Heinrichs, Quantum transport in a time-dependent random potential with a finite correlation time, Z. Phys. B 102 (1996) 137-144

M. Hillary, R.F. O'Connell, M.O. Scully, E.P. Wigner, Distribution functions in physics: fundamentals, Phys. Rep. 106 (1984) 121-167

W. Horsthemke, R. Lefever, Noise-induced transitions, theory and applications in Physics, Chemistry, and Biology, Springer, Berlin 1984

Y. Inaba, Diffusion of a quantum particle in a fluctuating environment, J. Phys. Soc. Japan 50 (1981) 2473-2480

V. Jakšić, C.-A. Pillet, Spectral theory of thermal relaxation, J. Math. Phys. 38 (1997a) $1757-1780$

V. Jakšić, C.-A. Pillet, Ergodic properties of the non-Markovian Langevin equation, Lett. Math. Phys. 41 (1997b) 49-57

A.P. Jauho, A model of dynamical disorder in a uniform time-dependent electric field, J. Phys. A 20 (1987) 2895-2902

A.M. Jayannavar, Wave propagation and quantum superdiffusion in a rapidly varying random potential, Phys. Rev. E 48 (1993) 837-842

A.M. Jayannavar, N. Kumar, Nondiffusive quantum transport in a dynamically disordered medium, Phys. Rev. Lett. 48 (1982) 553-556

E. Joos, Comment on "Unified dynamics for microscopic and macroscopic systems", Phys. Rev. D 36 (1987) 3285-3286

E. Joos, H.D. Zeh, The emergence of classical properties through interaction with the environment, Z. Phys. B 59 (1985) 223-243

G. Jumarie, Relative information, Springer, Berlin 1990

I. Karatzas, S.E. Shreve, Brownian motion and stochastic calculus, Springer, Berlin 1988

P. Kasperkovitz, M. Peev, Wigner-Weyl formalism for toroidal geometries, Ann. Phys. (N.Y.) 230 (1994) 21-51

K. Kitahara, J.W. Haus, Memory effects on the quantum diffusion of a particle in a fluctuating medium, Z. Phys. B 32 (1979) 419-423

R. Kubo, Generalized cumulant expansion method, J. Phys. Soc. Japan 17 (1962) 11001120

R. Kubo, Stochastic Liouville equation, J. Math. Phys. 4 (1963) 174-183

R. Kubo, M. Toda, N. Hashitsume, Statistical physics II: nonequilibrium statistical mechanics, 2nd ed., Springer, Berlin 1991. Japanese original: Iwanami Shoten, Tokyo 1978

W. Kundt, Classical statistics as a limiting case of quantum statistics, Z. Naturforschg. 22a (1967) 1333-1336

A. Lasota, M.C. Mackey, Chaos, fractals, and noise: stochastic aspects of dynamics, 2nd ed., Springer, New York 1994

N. Lebedev, P. Maass, S. Feng, Diffusion and superdiffusion of a particle in a random potential with finite correlation time, Phys. Rev. Lett. 74 (1995) 1895-1899

E.H. Lieb, Integral bounds for radar ambiguity functions and Wigner distributions, J. Math. Phys. 31 (1990) 594-599

I.M. Lifshits, S.A. Gredeskul, L.A. Pastur, Introduction to the theory of disordered systems, Wiley, New York 1988. Russian original: Nauka, Moscow 1982

G. Lindblad, On the generators of quantum dynamical semigroups, Commun. Math. Phys. 48 (1976) 119-130

H. Maassen, Return to thermal equilibrium by the solution of a quantum Langevin equation, J. Stat. Phys. 34 (1984) 239-261

A. Madhukar, W. Post, Exact solution for the diffusion of a particle in a medium with site diagonal and off-diagonal dynamic disorder, Phys. Rev. Lett. 39 (1977) 1424-1427

V.B. Magalinskiur, Dynamical model in the theory of the Brownian motion, Sov. Phys.JETP 9 (1959) 1381-1382. Russian original: Zh. Eksp. Teor. Fiz. 36 (1959) 1942-1944

P.A. Markowich, C.A. Ringhofer, C. Schmeiser, Semiconductor equations, Springer, Wien 1990 
F.H. Molzahn, T.A. Osborn, A phase space fluctuation method for quantum dynamics, Ann. Phys. (N.Y.) 230 (1994) 343-394

J.E. Moyal, Quantum mechanics as a statistical theory, Proc. Cambridge Philos. Soc. 45 (1949) 99-124

F.J. Narcowich, R.F. O'Connell, Necessary and sufficient conditions for a phase-space function to be a Wigner distribution, Phys. Rev. A 34 (1986) 1-6

P. Neu, R. Speicher, Spectra of Hamiltonians with generalized single-site dynamical disorder, Z. Phys. B 95 (1994) 101-111

E.A. Novikov, Functionals and the random-force method in turbulence theory, Sov. Phys.JETP 20 (1965) 1290-1294. Russian original: Zh. Eksp. Teor. Fiz. 47 (1964) 1919-1926

T.A. Osborn, F.H. Molzahn, Moyal quantum mechanics: the semiclassical Heisenberg dynamics, Ann. Phys. (N.Y.) 231 (1995) 79-127

A.A. Ovchinnikov, N.S. Érikhman, Motion of a quantum particle in a stochastic medium, Sov. Phys.-JETP 40 (1975) 733-737. Russian original: Zh. Eksp. Teor. Fiz. 67 (1974) $1474-1483$

L. Pastur, A. Figotin, Spectra of random and almost-periodic operators, Springer, Berlin 1992

P. Pearle, E. Squires, Bound state excitation, nucleon decay experiments, and models of wave function collapse, Phys. Rev. Lett. 73 (1994) 1-5

C.-A. Pillet, Some results on the quantum dynamics of a particle in a Markovian potential, Commun. Math. Phys. 102 (1985) 237-254

C.-A. Pillet, Asymptotic completeness for a quantum particle in a Markovian short-range potential, Commun. Math. Phys. 105 (1986) 259-280

J.C.T. Pool, Mathematical aspects of the Weyl correspondence, J. Math. Phys. 7 (1966) 66-76

M. Reed, B. Simon, Methods of modern mathematical physics III: scattering theory, Academic, New York 1979

S.L. Robinson, Semiclassical mechanics for time-dependent Wigner functions, J. Math. Phys. 34 (1993) 2185-2205

M.N. Rosenbluth, Comment on "Classical and quantum superdiffusion in a time-dependent random potential", Phys. Rev. Lett. 69 (1992) 1831

S.M. Rytov, Yu.A. Kravtsov, V.I. Tatarskiı̌, Principles of statistical radiophysics, vol. 3, Springer, Berlin 1989. Russian original: 2nd ed., Nauka, Moscow 1978

L. Saul, M. Kardar, N. Read, Directed waves in random media, Phys. Rev. A 45 (1992) 8859-8866

Yu.M. Shirokov, Quantum and classical mechanics in the phase space representation, Sov. J. Part. Nucl. 10 (1979) 1-18. Russian original: Fiz. Elem. Chastits At. Yadra 10 (1979) 5-50

B.I. Shklovskii, A.L. Efros, Electronic properties of doped semiconductors, Springer, Berlin 1984. Russian original: Nauka, Moscow 1979

K. Sogo, Y. Fujimoto, Quantum distribution function of a nonequilibrium system, Physica A 168 (1990) 820-832

H. Spohn, Kinetic equations from Hamiltonian dynamics: Markovian limits, Rev. Mod. Phys. 52 (1980) 569-615

S. Stintzing, Quantenmechanik eines Teilchens in einem zeitabhängigen Zufallspotential, Diploma Thesis, Ludwig-Maximilians-Universitt Mnchen 1992 (in German)

R.S. Streater, Statistical mechanics: a stochastic approach to nonequilibrium thermodynamics, Imperial College Press, London 1995

W. Sutherland, A dynamical theory of diffusion for non-elecrolytes and the molecular mass of albumin, Phil. Mag. (6th Series) 9 (1905) 781-785

V.I. Tatarskiú, The Wigner representation of quantum mechanics, Sov. Phys. Usp. 26 (1983) 311-327. Russian original: Usp. Fiz. Nauk. 139 (1983) 587-619

S. Tcheremchantsev, Markovian Anderson model: bounds for the rate of propagation, Commun. Math. Phys. 187 (1997) 441-469

W. Thirring, A course in mathematical physics 4: quantum mechanics of large systems, Springer, Wien 1983. German original: Springer, Wien 1980

P. Ullersma, An exactly solvable model for Brownian motion I-IV, Physica 32 (1966) $27-55,56-73,74-89,90-96$

U. Weiss, Quantum dissipative systems, World Scientific, Singapore 1993

R.F. Werner, The classical limit of quantum theory, preprint quant-ph/9504016, submitted 
to J. Math. Phys., 1995

H. Weyl, Quantenmechanik und Gruppentheorie, Z. Phys. 46 (1928) 1-46

E.P. Wigner, On the quantum correction for thermodynamic equilibrium, Phys. Rev. 40 (1932) 749-759

E.P. Wigner, Quantum-mechanical distribution functions revisited, in W. Yourgrau, A. van der Merwe (eds.), Perspectives in quantum theory, The M.I.T. Press, Cambridge (Mass.) 1971 , pp. 25-36

W.H. Zurek, Decoherence and the transition from quantum to classical, Physics Today, October 1991, 36-44. Commented on by J.L. Anderson; G.C. Ghirardi, R. Grassi, P. Pearle; N. Gisin; D.Z. Albert, G. Feinberg; P.R. Holland; V. Ambegaokar; K.J. Epstein, Physics Today, April 1993, 13-84. Reply to Comments, Physics Today, April 1993, 84-90 